\title{
SCHRÖDINGER EQUATIONS IN NONCYLINDRICAL DOMAINS: EXACT CONTROLLABILITY
}

\author{
G. O. ANTUNES, M. D. G. DA SILVA, AND R. F. APOLAYA \\ Received 6 July 2005; Accepted 12 March 2006
}

We consider an open bounded set $\Omega \subset \mathbb{R}^{n}$ and a family $\{K(t)\}_{t \geq 0}$ of orthogonal matrices of $\mathbb{R}^{n}$. Set $\Omega_{t}=\left\{x \in \mathbb{R}^{n} ; x=K(t) y\right.$, for all $\left.y \in \Omega\right\}$, whose boundary is $\Gamma_{t}$. We denote by $\hat{Q}$ the noncylindrical domain given by $\widehat{Q}=\bigcup_{0<t<T}\left\{\Omega_{t} \times\{t\}\right\}$, with the regular lateral boundary $\hat{\Sigma}=\bigcup_{0<t<T}\left\{\Gamma_{t} \times\{t\}\right\}$. In this paper we investigate the boundary exact controllability for the linear Schrödinger equation $u^{\prime}-i \Delta u=f$ in $\hat{Q}\left(i^{2}=-1\right), u=w$ on $\hat{\Sigma}$, $u(x, 0)=u_{0}(x)$ in $\Omega_{0}$, where $w$ is the control.

Copyright (c) 2006 Hindawi Publishing Corporation. All rights reserved.

\section{Introduction}

We consider the linear Schrödinger equation in a domain whose boundary is moving in time. Let $T$ be a positive real number and let $\left\{\Omega_{t}\right\}_{t \in[0, T]}$ be a family of bounded open sets of $\mathbb{R}^{n}$, with regular boundary $\Gamma_{t}$, defined as below. We denote by $\hat{Q}$ the noncylindrical domain of $\mathbb{R}^{n+1}$ defined by

$$
\widehat{Q}=\bigcup_{0<t<T}\left\{\Omega_{t} \times\{t\}\right\}
$$

with regular lateral boundary

$$
\hat{\Sigma}=\bigcup_{0<t<T}\left\{\Gamma_{t} \times\{t\}\right\}
$$

Let us consider $K$ a function such that for each $t \in[0, \infty)$, it associates an orthogonal matrix $K(t)=\left(a_{i j}(t)\right)_{n \times n}$. Note that $K^{-1}(t)=\left(a_{j i}(t)\right)_{n \times n}$. We denote $\left(a_{j i}^{\prime}(t)\right)_{n \times n}$ by $\left(K^{-1}\right)^{\prime}(t)$.

Let $\Omega$ be a bounded open set of $\mathbb{R}^{n}$, with regular boundary $\Gamma$. We consider the subsets $\Omega_{t}$ of $\mathbb{R}^{n}$ defined by

$$
\Omega_{t}=\left\{x \in \mathbb{R}^{n} ; x=K(t) y, y \in \Omega\right\}, \quad 0 \leq t \leq T .
$$


2 Schrödinger equations in noncylindrical domains

We develop the article under the following assumptions:

(H1) $K \in C^{2}[0, T]$;

(H2) there exists a constant $\alpha>0$ such that

$$
\left(\left(K^{-1}\right)^{\prime} K\right) w \cdot w \geq \alpha|w|^{2} \quad \forall w \in \mathbb{C}^{n} .
$$

The aim of this paper is to obtain the exact controllability of the following mixed problem:

$$
\mid \begin{aligned}
& u^{\prime}-i \Delta u=\hat{f} \quad \text { in } \hat{Q}, \\
& u=\mid \begin{array}{cc}
\hat{\varphi} & \text { on } \hat{\Sigma}_{0} \\
0 & \text { on } \hat{\Sigma} \backslash \hat{\Sigma}_{0}
\end{array} \quad\left(i^{2}=-1\right), \\
& u(x, 0)=u_{0}(x) \quad \text { in } \Omega_{0}
\end{aligned}
$$

where $\hat{\Sigma}_{0}$ is a part of $\hat{\Sigma}$ with positive measure.

We can formulate the exact controllability problem for (1.5) as follows: given $T>0$ large enough, we want to find a Hilbert space $H$ such that, for each initial data $u_{0}$ belonging to $H$, there exists a control $\hat{\varphi}$ belonging to the space of controls, defined on $\hat{\Sigma}_{0}$, such that a solution $u=u(x, t)$ of (1.5) satisfies the final condition

$$
u(x, T)=0 \quad \text { in } \Omega_{T} .
$$

The methodology (cf. Lions [7]) consists of transforming (1.5) into an equivalent problem in the cylinder $Q=\Omega \times(0, T)$ by the diffeomorphism

$$
\tau: \hat{Q} \longrightarrow Q
$$

defined by $\tau(x, t)=(y, t)$, with $y=K^{-1}(t) x$. The inverse

$$
\tau^{-1}: Q \longrightarrow \hat{Q}
$$

is defined by $\tau^{-1}(y, t)=(K(t) y, t)$. Then by the change of variables $u(x, t)=v(y, t)$, where $y=K^{-1}(t) x, y \in \Omega$, and $x \in \Omega_{t}$, we obtain

$$
\begin{gathered}
u^{\prime}(x, t)=v^{\prime}(y, t)+\nabla v \cdot\left(K^{-1}(t)\right)^{\prime} K(t) y, \\
\Delta u(x, t)=\Delta v(y, t) .
\end{gathered}
$$

Therefore, we transform the problem (1.5) in the noncylindrical domain $\widehat{Q}$ into the following problem in the cylinder $Q$ :

$$
\mid \begin{aligned}
& v^{\prime}-i \Delta v+\nabla v \cdot\left(K^{-1}\right)^{\prime} K y=f \quad \text { in } Q, \\
& v(y, t)=\mid \begin{array}{cc}
\varphi & \text { on } \Sigma_{0} \\
0 & \text { on } \Sigma \backslash \Sigma_{0}
\end{array} \quad\left(i^{2}=-1\right), \\
& v(y, 0)=v_{0}(y) \quad \text { in } \Omega,
\end{aligned}
$$


where $\Sigma=\Gamma \times(0, T)$ is the lateral boundary of the cylinder $Q$ and $\Sigma_{0}$ is a part of $\Sigma$, that will be defined in Section 5 .

We investigate the exact controllability for the equivalent problem (1.10) using HUM (hilbert uniqueness method) idealized by Lions [8]. The particular case where $\tau_{t}(y)=$ $\mu(t) y$, with $\mu(t)$ a real function defined on nonnegative real numbers $[0, \infty)$, has been analyzed by Miranda and Medeiros [10]. We can also find a study in controllability for Schrödinger equation in cylindrical domains in Lebeau [6] and Machtyngier [9]. We include, in the references at the end of this paper, some works relating to noncylindrical mixed problems for others models and related arguments, such as: [2-5].

The plan of this paper is as follows. In Section 2, we study the properties of the weak solution of the homogeneous boundary value problem for the formal adjoint $L^{*}$ of the operator of (1.10), which is calculated in Section 4. Section 3 is dedicated to prove the direct and inverse inequalities for the weak solutions obtained in Section 2. Section 5 is dedicated to solve the problem of exact controllability for (1.10) and (1.5), respectively. In the appendix, we prove an identity which is a key point for the second estimate in Section 2. Below we state the main result of our paper.

Theorem 1.1. Let $\Omega$ be a regular, bounded open set of $\mathbb{R}^{n}$, let $\Omega_{t}$ be defined as in (1.3), and suppose that (H1) and (H2) hold. If $T>0$, then, for each $u_{0} \in H^{-1}\left(\Omega_{t}\right)$, there exists a control $\widehat{w} \in L^{2}(\hat{\Sigma})$ such that $u$ solution of problem (1.5) verifies

$$
u(x, T, \widehat{w})=0 \quad \forall x \in \Omega_{T} .
$$

\section{Weak solutions}

The formal adjoint or transposed operator of $L w=w^{\prime}-i \Delta w+\nabla w \cdot\left(K^{-1}\right)^{\prime} K y$ is $-L^{*} v=$ $v^{\prime}-i \Delta v+\nabla v \cdot\left(K^{-1}\right)^{\prime} K y+\operatorname{tr}\left(\left(K^{-1}\right) K\right) v$. We represent by $C(t)=\left(c_{j k}(t)\right)_{1 \leq j, k \leq n}$ the ma$\operatorname{trix}\left(K^{-1}(t)\right)^{\prime} K(t)$ and by $\operatorname{tr}(C(t))$ its trace.

By $H^{1}(\Omega)$ we represent the Sobolev space defined by

$$
\left\{u \in L^{2}(\Omega), \frac{\partial u}{\partial x_{i}} \in L^{2}(\Omega) \text { for } i=1, \ldots, n\right\}
$$

Also by $H_{0}^{1}(\Omega)$ we represent the Sobolev space of functions $u \in H^{1}(\Omega)$ such that $\left.u\right|_{\Gamma}=0$.

In order to apply HUM to the mixed problem (1.10) it is fundamental to know the properties of the weak solution of the homogeneous boundary value problem for the formal adjoint $L^{*}$, which is studied in this section.

We consider the following problem for the adjoint $L^{*}$.

Given $v_{0} \in H_{0}^{1}(\Omega)$ and $f \in L^{2}\left(0, T ; H_{0}^{1}(\Omega)\right)$, we want to find a function $v: Q \rightarrow \mathbb{C}$ solution, in some sense, of the boundary value problem

$$
\mid \begin{aligned}
& v^{\prime}-i \Delta v+\nabla v \cdot C y+\operatorname{tr}(C) v=f \quad \text { in } Q, \\
& v=0 \quad \text { on } \Sigma, \\
& v(0)=v_{0} \quad \text { in } \Omega .
\end{aligned}
$$


THEOREM 2.1. Given $v_{0} \in H_{0}^{1}(\Omega)$ and $f \in L^{2}\left(0, T ; H_{0}^{1}(\Omega)\right)$, there exists one and only one function $v: Q \rightarrow \mathbb{C}$, called the weak solution of (2.2), satisfying

$$
\begin{gathered}
v \in L^{\infty}\left(0, T ; H_{0}^{1}(\Omega)\right) \\
v^{\prime} \in L^{2}\left(0, T ; H^{-1}(\Omega)\right), \\
-\int_{0}^{T}\left(v, \varphi^{\prime}\right) d t+i \int_{0}^{T}((v, \varphi)) d t+\int_{0}^{T}(\nabla v \cdot C(t) y, \varphi) d t+\int_{0}^{T}(\operatorname{tr}(C) v, \varphi) d t=\int_{0}^{T}(f, \varphi) d t
\end{gathered}
$$

for all $\varphi \in L^{2}\left(0, T ; H_{0}^{1}(\Omega)\right)$, such that $\varphi^{\prime} \in L^{2}\left(0, T ; L^{2}(\Omega)\right)$ with $\varphi(0)=\varphi(T)=0$,

$$
v(0)=v_{0} \quad \text { in } \Omega \text {. }
$$

Observe that $(\cdot, \cdot)$ and $|\cdot|,((\cdot, \cdot))$ and $\|\cdot\|$ represent the inner product and norm, respectively, in $L^{2}(\Omega)$ and $H_{0}^{1}(\Omega)$.

Proof. We employ the Galerkin method. In fact, let us consider the sequence $\left(w_{j}\right)_{j \in \mathbb{N}}$ of the solutions of the eigenvalue problem

$$
\left(\left(w_{j}, \varphi\right)\right)=\lambda_{j}\left(w_{j}, \varphi\right), \quad j=1,2, \ldots,
$$

for each $\varphi \in H_{0}^{1}(\Omega)$. Represent by $V_{m}$ the subspace generated by $\left\{w_{1}, w_{2}, \ldots, w_{m}\right\}$ and let us consider the approximate problem

$$
\mid \begin{array}{r}
\text { find } v_{m} \in V_{m} \text { solution of }\left(\begin{array}{l}
\left.v_{m}^{\prime}(t), w_{j}\right)+i\left(\left(v_{m}(t), w_{j}\right)\right)+\left(\nabla v_{m}(t) \cdot C(t) y, w_{j}\right) \\
+\operatorname{tr}(C(t))\left(v_{m}(t), w_{j}\right)=\left(f(t), w_{j}\right) \quad \text { for } j=1,2, \ldots, m,
\end{array}\right. \\
v_{m}(0)=v_{0 m} \text { strongly convergent to } v_{0} \text { in } H_{0}^{1}(\Omega) .
\end{array}
$$

Note that if $v_{m}(t) \in V_{m}$, then $v_{m}(y, t)=\sum_{j=1}^{m} g_{j m}(t) w_{j}(y)$. It follows that (2.6) is a system of ordinary differential equations in the unknowns $g_{j m}(t), j=1,2, \ldots, m$. This system has a local solution on $\left[0, t_{m}\right)$, for some $t_{m} \in(0, T)$, and each $g_{j m}(t)$ belongs to $H^{1}\left(0, t_{m}\right)$. The extension to interval $[0, T]$ is a consequence of the following estimate.

First estimate. Multiply both sides of (2.6) by $\overline{g_{j m}(t)}$, adding from $j=1$ to $j=m$, we obtain

$$
\begin{gathered}
\left(v_{m}^{\prime}(t), v_{m}(t)\right)+i\left(\left(v_{m}(t), v_{m}(t)\right)\right)+\left(\nabla v_{m}(t) \cdot C(t) y, v_{m}(t)\right) \\
+\operatorname{tr}(C(t))\left(v_{m}(t), v_{m}(t)\right)=\left(f(t), v_{m}(t)\right) .
\end{gathered}
$$

Note that $\bar{z}$ is the complex conjugate of $z$.

Taking the double of the real parts of the last equality's both sides, we obtain

$$
\begin{aligned}
2 \operatorname{Re} & \left(v_{m}^{\prime}(t), v_{m}(t)\right)+2 \operatorname{Re}\left(\nabla v_{m}(t) \cdot C(t) y, v_{m}(t)\right)+2 \operatorname{tr}(C(t))\left|v_{m}(t)\right|^{2} \\
& =2 \operatorname{Re}\left(f(t), v_{m}(t)\right) .
\end{aligned}
$$

Observe that $\nabla v_{m}(t) \cdot y=\left(\partial v_{m}(t) / \partial y_{j}\right) y_{j}$ and repeated indexes mean summation. 
We analyze the first and second terms of the last equality.

(i) $2 \operatorname{Re}\left(v_{m}^{\prime}(t), v_{m}(t)\right)$.

We have

$$
2 \operatorname{Re}\left(v_{m}^{\prime}(t), v_{m}(t)\right)=\frac{d}{d t}\left|v_{m}(t)\right|^{2} .
$$

(ii) $2 \operatorname{Re}\left(\nabla v_{m}(t) \cdot C(t) y, v_{m}(t)\right)$.

By Gauss' lemma,

$$
\int_{\Omega} \frac{\partial}{\partial y_{j}}\left(v_{m}(t) \mathcal{c}_{j k}(t) y_{k} \overline{v_{m}(t)}\right) d y=0
$$

We observe that

$$
\frac{\partial}{\partial y_{j}}\left(c_{j k}(t) y_{k}\right)=c_{j j}(t) y_{j}
$$

and therefore

$$
\int_{\Omega} \frac{\partial v_{m}(t)}{\partial y_{j}} c_{j k}(t) y_{k} \overline{v_{m}(t)} d y+\int_{\Omega} v_{m}(t) c_{j j}(t) \overline{v_{m}(t)} d y+\int_{\Omega} v_{m}(t) c_{j k}(t) y_{k} \frac{\overline{\partial v_{m}(t)}}{\partial y_{j}} d y=0 .
$$

Thus,

$$
2 \operatorname{Re}\left(\nabla v_{m}(t) \cdot C(t) y, v_{m}(t)\right)=-\sum_{j=1}^{n} \int_{\Omega} c_{j j}(t)\left|v_{m}(t, y)\right|^{2} d y=-\operatorname{tr}(C)\left|v_{m}(t)\right|^{2} .
$$

Substituting (2.9) and (2.13) in (2.8), it follows that

$$
\frac{d}{d t}\left|v_{m}(t)\right|^{2}+\operatorname{tr}(C)\left|v_{m}(t)\right|^{2} \leq 2|f(t)|\left|v_{m}(t)\right| .
$$

From assumption $(\mathrm{H} 2)$ that $\operatorname{tr}(C(t)) \geq 0$, it follows that

$$
\frac{d}{d t}\left|v_{m}(t)\right|^{2} \leq 2|f(t)|\left|v_{m}(t)\right|
$$

that is,

$$
\frac{d}{d t}\left(\left|v_{m}(t)\right|^{2}\right)-\left|v_{m}(t)\right|^{2} \leq|f(t)|^{2} .
$$

Multiplying (2.16) by $e^{-t}$ and integrating from 0 to $t$ we obtain

$$
\left|v_{m}(t)\right|^{2} \leq C_{1}\left\{\left|v_{0 m}\right|^{2}+\|f\|_{L^{2}\left(0, T ; L^{2}(\Omega)\right)}^{2}\right\} .
$$

Since $v_{m}(0)=v_{0 m} \rightarrow v_{0}$ in $H_{0}^{1}(\Omega)$, we conclude that

$$
\left(v_{m}(t)\right) \text { is bounded in } L^{\infty}\left(0, T ; L^{2}(\Omega)\right) .
$$


Second estimate. Multiply both sides of (2.6) by $\lambda_{j} \overline{g_{j m}(t)}$, adding from $j=1$ to $j=m$ and taking the double of real parts, we obtain

$$
\begin{aligned}
& 2 \operatorname{Re}(\left.v_{m}^{\prime}(t),-\Delta v_{m}(t)\right)+2 \operatorname{Re}\left(\nabla v_{m}(t) \cdot C(t) y,-\Delta v_{m}(t)\right) \\
& \quad+2 \operatorname{tr}(C(t))\left(v_{m}(t),-\Delta v_{m}(t)\right)=2 \operatorname{Re}\left(f(t),-\Delta v_{m}(t)\right) .
\end{aligned}
$$

(i) Analysis of $2 \operatorname{Re}\left(v_{m}^{\prime}(t),-\Delta v_{m}(t)\right)$.

We have,

$$
2 \operatorname{Re}\left(v_{m}^{\prime}(t),-\Delta v_{m}(t)\right)=2 \operatorname{Re}\left(\left(v_{m}^{\prime}(t), v_{m}(t)\right)\right)=\frac{d}{d t}\left\|v_{m}(t)\right\|^{2}
$$

(ii) Analysis of $2 \operatorname{Re}\left(\nabla v_{m}(t) \cdot C(t) y,-\Delta v_{m}(t)\right)$.

By Green's formula,

$$
\begin{aligned}
\left(\frac{\partial v_{m}(t)}{\partial y_{j}} c_{j k}(t) y_{k},-\Delta v_{m}(t)\right) & \\
= & \left(\frac{\partial}{\partial y_{l}}\left[\frac{\partial v_{m}(t)}{\partial y_{j}} c_{j k}(t) y_{k}\right], \frac{\partial v_{m}(t)}{\partial y_{l}}\right)-\int_{\Gamma} c_{j k} y_{k} \cdot v_{j}\left|\frac{\partial v_{m}}{\partial \nu}\right|^{2} d \Gamma \\
= & \left(\frac{\partial}{\partial y_{l}}\left(\frac{\partial v_{m}(t)}{\partial y_{j}}\right) c_{j k}(t) y_{k}, \frac{\partial v_{m}(t)}{\partial y_{l}}\right)+\left(\frac{\partial v_{m}(t)}{\partial y_{j}} c_{j k}(t) \delta_{l}^{k}, \frac{\partial v_{m}(t)}{\partial y_{l}}\right) \\
& -\int_{\Gamma} c_{j k} y_{k} \cdot v_{j}\left|\frac{\partial v_{m}}{\partial \nu}\right|^{2} d \Gamma=\int_{\Omega} \frac{\partial}{\partial y_{l}}\left(\frac{\partial v_{m}(t)}{\partial y_{j}}\right) c_{j k}(t) y_{k} \frac{\partial v_{m}(t)}{\partial y_{l}} d y \\
& +\int_{\Omega} \frac{\partial v_{m}(t)}{\partial y_{j}} c_{j l}(t) \frac{\partial v_{m}(t)}{\partial y_{l}} d y-\int_{\Gamma} c_{j k} y_{k} \cdot v_{j}\left|\frac{\partial v_{m}}{\partial \nu}\right|^{2} d \Gamma .
\end{aligned}
$$

It follows from (2.21) that

$$
\begin{aligned}
2 \operatorname{Re}( & \left.\frac{\partial v_{m}(t)}{\partial y_{j}} c_{j k}(t) y_{k},-\Delta v_{m}(t)\right) \\
= & 2 \operatorname{Re}\left(\frac{\partial}{\partial y_{l}}\left(\frac{\partial v_{m}(t)}{\partial y_{j}}\right) c_{j k}(t) y_{k}, \frac{\partial v_{m}(t)}{\partial y_{l}}\right) \\
& +2 \operatorname{Re}\left(\frac{\partial v_{m}(t)}{\partial y_{j}} c_{j l}(t), \frac{\partial v_{m}(t)}{\partial y_{l}}\right)-2 \int_{\Gamma} c_{j k} y_{k} \cdot v_{j}\left|\frac{\partial v_{m}}{\partial v}\right|^{2} d \Gamma .
\end{aligned}
$$

Now, by Gauss' lemma,

$$
\int_{\Omega} \frac{\partial}{\partial y_{j}}\left(\frac{\partial v_{m}(t)}{\partial y_{l}} c_{j k}(t) y_{k} \frac{\overline{\partial v_{m}(t)}}{\partial y_{l}}\right) d y=\int_{\Gamma} v_{j} \cdot\left(\frac{\partial v_{m}(t)}{\partial y_{l}} c_{j k}(t) y_{k} \frac{\overline{\partial v_{m}(t)}}{\partial y_{l}}\right) d \Gamma
$$


That is,

$$
\begin{aligned}
& \int_{\Omega} \frac{\partial}{\partial y_{j}}\left(\frac{\partial v_{m}(t)}{\partial y_{l}}\right) c_{j k}(t) y_{k} \frac{\overline{\partial v_{m}(t)}}{\partial y_{l}} d y \\
& +\int_{\Omega} \frac{\partial v_{m}(t)}{\partial y_{l}} c_{j j}(t) \frac{\overline{\partial v_{m}(t)}}{\partial y_{l}} d y+\int_{\Omega} \frac{\partial v_{m}(t)}{\partial y_{l}} c_{j k}(t) y_{k} \overline{\frac{\partial}{\partial y_{j}}\left(\frac{\partial v_{m}(t)}{\partial y_{l}}\right)} d y \\
& =\int_{\Gamma} v_{j} \cdot\left(\frac{\partial v_{m}(t)}{\partial y_{l}} c_{j k}(t) y_{k} \frac{\overline{\partial v_{m}(t)}}{\partial y_{l}}\right) d \Gamma=\int_{\Gamma} v_{j} c_{j k}(t) y_{k}\left|\frac{\partial v_{m}}{\partial \nu}\right|^{2} d \Gamma .
\end{aligned}
$$

Therefore,

$$
2 \operatorname{Re}\left(\frac{\partial}{\partial y_{j}}\left(\frac{\partial v_{m}(t)}{\partial y_{l}}\right) c_{j k}(t) y_{k}, \frac{\partial v_{m}(t)}{\partial y_{l}}\right)=\int_{\Gamma} v_{j} c_{j k}(t) y_{k}\left|\frac{\partial v_{m}}{\partial \nu}\right|^{2} d \Gamma-c_{j j}(t) \int_{\Omega}\left|\frac{\partial v_{m}}{\partial y_{l}}\right|^{2} d y .
$$

Substituting (2.25) into (2.22), we obtain

$$
\begin{aligned}
& 2 \operatorname{Re}\left(\frac{\partial v_{m}(t)}{\partial y_{j}} c_{j k}(t) y_{k},-\Delta v_{m}(t)\right) \\
& \quad=2 \operatorname{Re}\left(\frac{\partial v_{m}(t)}{\partial y_{j}} c_{j l}(t), \frac{\partial v_{m}(t)}{\partial y_{l}}\right)-c_{j j}(t) \int_{\Omega}\left|\frac{\partial v_{m}}{\partial y_{l}}\right|^{2} d y-\int_{\Gamma} v_{j} c_{j k}(t) y_{k}\left|\frac{\partial v_{m}}{\partial \nu}\right|^{2} d \Gamma .
\end{aligned}
$$

Substituting the expressions (2.20) and (2.26) into (2.19) we obtain

$$
\begin{aligned}
& \frac{d}{d t} \mid\left.v_{m}(t)\right|^{2}+2 \operatorname{Re}\left(\frac{\partial v_{m}(t)}{\partial y_{j}} c_{j l}(t), \frac{\partial v_{m}(t)}{\partial y_{l}}\right)-c_{j j}(t) \int_{\Omega}\left|\frac{\partial v_{m}}{\partial y_{l}}\right|^{2} d y \\
&-\int_{\Gamma} v_{j} c_{j k}(t) y_{k}\left|\frac{\partial v_{m}}{\partial \nu}\right|^{2} d \Gamma+2 \operatorname{tr}(C(t))\left\|v_{m}(t)\right\|^{2}=2 \operatorname{Re}\left(f(t),-\Delta v_{m}(t)\right) .
\end{aligned}
$$

By the identity of the appendix, we modify (2.27) as follows:

$$
\begin{aligned}
\frac{d}{d t}\left\|v_{m}(t)\right\|^{2}+\operatorname{tr}(C(t))\left\|v_{m}(t)\right\|^{2}-\frac{d}{d t} \operatorname{Im}\left(v_{m}(t), C(t) y \cdot \nabla v_{m}(t)\right) \\
\quad+\operatorname{Im}\left(v_{m}(t), C^{\prime}(t) y \cdot \nabla v_{m}(t)\right)-\operatorname{tr}(C(t)) \operatorname{Im}\left(v_{m}(t), C(t) y \cdot \nabla v_{m}(t)\right) \\
\quad+2 \operatorname{Im}\left(P_{m} f(t), C(t) y \cdot \nabla v_{m}(t)\right)+\operatorname{tr}(C(t)) \operatorname{Im}\left(P_{m} f(t), v_{m}(t)\right) \\
=2 \operatorname{Re}\left(\left(f(t), v_{m}(t)\right)\right) .
\end{aligned}
$$

Setting $\varphi(t)=\operatorname{Im}\left(v_{m}(t), C(t) y \cdot \nabla v_{m}(t)\right)$ and $\psi(t)=\left\|v_{m}(t)\right\|^{2}-\varphi(t)$, we obtain from (2.28) that

$$
\frac{d}{d t} \psi(t)+\operatorname{tr}(C(t)) \psi(t)=g(t)
$$


8 Schrödinger equations in noncylindrical domains

where

$$
\begin{aligned}
g(t)= & 2 \operatorname{Re}\left(\left(f(t), v_{m}(t)\right)\right)-\operatorname{tr}(C(t)) \operatorname{Im}\left(f(t), v_{m}(t)\right) \\
& -2 \operatorname{Im}\left(P_{m} f(t), C(t) y \cdot \nabla v_{m}(t)\right)-\operatorname{Im}\left(v_{m}(t), C^{\prime}(t) y \cdot \nabla v_{m}(t)\right) .
\end{aligned}
$$

Solving the differential equation (2.29) we attain

$$
\psi(t)=\psi(0) \exp \left(-\int_{0}^{t} \operatorname{tr}(C(r)) d r\right)+\int_{0}^{t} \exp \left(-\int_{s}^{t} \operatorname{tr}(C(r)) d r\right) g(s) d s
$$

Since $\psi(0)=\left\|v_{m}(0)\right\|^{2}-\varphi(0)$, we have from the last equality

$$
\begin{aligned}
\left\|v_{m}(t)\right\|^{2}= & \varphi(t)+\left(\left\|v_{m}(0)\right\|^{2}-\varphi(0)\right) \exp \left(-\int_{0}^{t} \operatorname{tr}(C(r)) d r\right) \\
& +\int_{0}^{t} \exp \left(-\int_{s}^{t} \operatorname{tr}(C(r)) d r\right) g(s) d s .
\end{aligned}
$$

Observe that

(i)

$$
\begin{aligned}
\varphi(t) & \leq\left|v_{m}(t)\right|\left|C(t) y \cdot \nabla v_{m}(t)\right| \leq\left|v_{m}(t)\right| \sum_{l=1}^{n}\left(\sum_{k=1}^{n}\left|c_{l k}(t)\right|\left|y_{k}\right|\right)\left|\frac{\partial v_{m}}{\partial y_{l}}\right| \\
& \leq\left|v_{m}(t)\right|\left[\sum_{l=1}^{n}\left(\sum_{k=1}^{n}\left|c_{l k}(t)\right|\left|y_{k}\right|\right)^{2}\right]^{1 / 2}\left(\sum_{l=1}^{n}\left|\frac{\partial v_{m}}{\partial y_{l}}\right|^{2}\right)^{1 / 2} \\
& \leq\left|v_{m}(t)\right|\left[\sum_{l=1}^{n}\left(\sum_{k=1}^{n}\left|c_{l k}(t)\right|^{2}\right)\left(\sum_{k=1}^{n}\left|y_{k}\right|^{2}\right)\right]^{1 / 2}\left(\sum_{l=1}^{n}\left|\frac{\partial v_{m}}{\partial y_{l}}\right|^{2}\right)^{1 / 2} \\
& =\left|v_{m}(t)\right||y|\left[\sum_{l=1}^{n} \sum_{k=1}^{n}\left|c_{l k}(t)\right|^{2}\right]^{1 / 2}\left|\nabla v_{m}\right| \\
& \leq\left|v_{m}(t)\right||y| n\left(\max _{l, k=1, \ldots, n}\left(\max _{t \in[0, T]}\left|c_{l k}(t)\right|\right)\right)\left|\nabla v_{m}\right| \\
& \leq M\left(\Omega, n, M_{C}\right)\left|v_{m}(t)\right|\left|\nabla v_{m}\right| \leq \frac{M\left(\Omega, n, M_{C}\right)^{2}}{2}\left|v_{m}(t)\right|^{2}+\frac{\left|\nabla v_{m}\right|^{2}}{2}
\end{aligned}
$$

where $M_{C}=\left(\max _{l, k=1, \ldots, n}\left(\max _{t \in[0, T]}\left|c_{l k}(t)\right|\right)\right)$ and $M\left(\Omega, n, M_{C}\right)=\left(\max _{y \in \Omega}|y|\right) n M_{C}$. Since (ii)

$$
\exp \left(-\int_{s}^{t} \operatorname{tr}(C(r)) d r\right) \leq \exp \left(\int_{s}^{t}|\operatorname{tr}(C(r))| d r\right) \leq \exp \left(n M_{C} T\right)
$$

(iii)

$$
|g(s)| \leq M\left(n, \Omega, M_{C}\right)\left(\|f(s)\|+\left|v_{m}(s)\right|\right)|| v_{m}(s) \|,
$$


G. O. Antunes et al. 9

we have

$$
\begin{aligned}
& \left|\int_{0}^{t} \exp \left(-\int_{s}^{t} \operatorname{tr}(C(r)) d r\right) g(s) d s\right| \\
& \quad \leq M\left(n, \Omega, M_{C}, T\right) \int_{0}^{t}\left(\| f(s)||+\left|v_{m}(s)\right|\right)\left\|v_{m}(s)\right\| d s,
\end{aligned}
$$

where

$$
M\left(n, \Omega, M_{C}, T\right)=\exp \left(n M_{C} T\right) M\left(n, \Omega, M_{C}\right)
$$

and therefore

$$
\begin{aligned}
\left\|v_{m}(t)\right\|^{2} \leq & \frac{1}{2} M\left(n, \Omega, M_{C}\right)\left|v_{m}(t)\right|^{2}+\frac{1}{2}\left\|v_{m}(t)\right\|^{2}+M\left(n, \Omega, M_{C}, T\right)\left\|v_{0 m}\right\|^{2} \\
& +M\left(n, \Omega, M_{C}, T\right) \int_{0}^{t}\left(\|f(s)\|+\left|v_{m}(s)\right|\right)\left\|v_{m}(s)\right\| d s .
\end{aligned}
$$

From the first estimate, it follows that

$$
\left|v_{m}(t)\right|^{2} \leq \alpha_{0}(T) .
$$

Then (2.39) implies

$$
\frac{\left\|v_{m}(t)\right\|^{2}}{2} \leq M\left(n, \Omega, M_{C}, T,\left\|v_{0 m}\right\|\right)+M\left(n, \Omega, M_{C}, T\right) \int_{0}^{t}\left(\|f(s)\|+\left|v_{m}(s)\right|\right)\left\|v_{m}(s)\right\| d s,
$$

where

$$
M\left(n, \Omega, M_{C}, T,\left\|v_{0 m}\right\|\right)=\frac{1}{2} M\left(n, \Omega, M_{C}\right) \alpha_{0}^{2}+M\left(n, \Omega, M_{C}, T\right)\left\|v_{0 m}\right\|^{2} .
$$

Then, by an inequality similar to Gronwall-Bellman's one, see Brézis [1, page 157], we obtain

$$
\left\|v_{m}(t)\right\|^{2} \leq \alpha_{1}(T)
$$

Then

$$
\left(v_{m}\right) \text { is bounded in } L^{\infty}\left(0, T ; H_{0}^{1}(\Omega)\right) \text {. }
$$

Then, from (2.43), we can extract a subsequence $v_{\mu}$ of $v_{m}$ such that

$$
v_{\mu} \text { converges to } v \text { weak star in } L^{\infty}\left(0, T ; H_{0}^{1}(\Omega)\right) .
$$

Applying the approximate equation (2.6) to $\theta \in \mathscr{D}(0, T)$, we get

$$
\begin{gathered}
\int_{0}^{T}\left(v_{\mu}, w_{j}\right) \theta^{\prime} d t+\int_{0}^{T} i\left(\left(v_{\mu}, w_{j}\right)\right) \theta d t+\int_{0}^{T}\left(C y \cdot \nabla v_{\mu}, w_{j}\right) \theta d t \\
+\int_{0}^{T} \operatorname{tr}(C(t))\left(v_{\mu}, w_{j}\right) \theta d t=\int_{0}^{T}\left(f, w_{j}\right) \theta d t
\end{gathered}
$$


Taking the limit when $\mu \rightarrow \infty, j$ fixed, we obtain that $v$ is a solution in the sense of Theorem 2.1.

We observe that

$$
\frac{d}{d t}(v(t), w)+i((v(t), w))+(C y \cdot \nabla v(t), w)+\operatorname{tr}(C(t))(v(t), w)=(f(t), w),
$$

in the sense of $\mathscr{D}^{\prime}(0, T)$, for each $w \in H_{0}^{1}(\Omega)$.

Since $G=i \Delta v-C y \cdot \nabla v-\operatorname{tr}(C) v+f \in L^{2}\left(0, T ; H^{-1}(\Omega)\right)$, it follows from (2.46) (cf. Temam [11]) that

$$
v^{\prime} \in L^{2}\left(0, T ; H^{-1}(\Omega)\right), \quad v^{\prime}=G \quad \text { in } L^{2}\left(0, T ; H^{-1}(\Omega)\right) .
$$

As we have seen $v \in L^{2}\left(0, T ; H_{0}^{1}(\Omega)\right)$, then, identifying $L^{2}(\Omega)$ to its dual $\left(L^{2}(\Omega)\right)^{\prime}$, we obtain

$$
v \in C^{0}\left([0, T] ; L^{2}(\Omega)\right) .
$$

The equality stated in Theorem 2.1 follows from the one in (2.47), as the uniqueness of solution does too. We must observe that the solution $v$ also satisfies $v(0)=v_{0}$.

To prove uniqueness, suppose $v_{1}, v_{2}$ are solutions and $w=v_{1}-v_{2}$. Then, by the equality in (2.47), we obtain

$$
w^{\prime}-i \Delta w+C y \cdot \nabla w+\operatorname{tr}(C) w=0 \quad \text { in } L^{2}\left(0, T ; H^{-1}(\Omega)\right) .
$$

Thus, multiplying by $w$ and taking the double of the real part of it, we have

$$
2 \operatorname{Re}\left\langle w^{\prime}(t), w(t)\right\rangle+2 \operatorname{Re}(\nabla w(t) \cdot C(t) y, w(t))+2 \operatorname{tr}(C(t))|w(t)|^{2}=0 .
$$

Analyzing the first and second terms of the last equality as in the first estimate, we attain

$$
\frac{d}{d t}|w(t)|^{2}+\operatorname{tr}(C(t))|w(t)|^{2} \leq 0
$$

From assumption $(\mathrm{H} 2)$ that $\operatorname{tr}(C(t)) \geq 0$ it follows that

$$
\frac{d}{d t}|w(t)|^{2} \leq 0
$$

and since $w(0)=0$, we conclude that $w \equiv 0$ which means the solution $v$ is unique.

Remark 2.2. If we consider the boundary value problem (2.2) with initial data

$$
v_{0} \in H_{0}^{1}(\Omega) \cap H^{2}(\Omega) \quad f \in L^{2}\left(0, T ; H_{0}^{1}(\Omega)\right) \quad \text { such that } f^{\prime} \in L^{1}\left(0, T ; L^{2}(\Omega)\right),
$$

by the same argument used to prove Theorem 2.1, we prove the following theorem. 
Theorem 2.3. Given $v_{0}$ and $f$ satisfying (2.53), there exists only one function $v: Q \rightarrow \mathbb{C}$, satisfying the following conditions:

$$
\mid \begin{aligned}
& v \in L^{\infty}\left(0, T ; H_{0}^{1}(\Omega) \cap H^{2}(\Omega)\right), \\
& v^{\prime}-i \Delta v+C y \cdot \nabla v+\operatorname{tr}(C) v=f \quad \text { a.e. in } Q, \\
& v(0)=v_{0} \quad \text { in } \Omega .
\end{aligned}
$$

The solution $v$ of Theorem 2.3 is called the strong solution of problem (2.2).

\section{Inequalities}

HUM is based on two inequalities: one is called direct, Lemma 3.2; another inverse, Lemma 3.3. This section is dedicated to prove these inequalities, for the solution of the boundary value problem (2.2), which are the key points in order to solve the exact controllability problem for the Schrödinger equation, see (5.19).

We begin proving the following identity.

Lemma 3.1. Let $q=\left(q_{l}\right), 1 \leq l \leq n$, be a vector field, with $q_{l} \in C^{2}(\bar{\Omega})$ for all $l$. Then, for all strong solution $v$ of the adjoint boundary value problem (2.2), there exists the identity

$$
\begin{aligned}
\int_{0}^{T} \int_{\Gamma} q \cdot v\left|\frac{\partial v}{\partial v}\right|^{2} d \Gamma d t \\
=\left.\operatorname{Im}\left(v, q_{l} \frac{\partial v}{\partial y_{l}}\right)\right|_{0} ^{T}+\int_{0}^{T} \operatorname{Re}\left(\frac{\partial v}{\partial y_{j}}, \frac{\partial^{2} q_{l}}{\partial y_{j} \partial y_{l}} v\right) d t \\
\quad+2 \operatorname{Re}\left(\frac{\partial v}{\partial y_{j}}, \frac{\partial q_{l}}{\partial y_{j}} \frac{\partial v}{\partial y_{l}}\right) d t+\int_{0}^{T} \operatorname{Im}\left(c_{j k} y_{k} \frac{\partial v}{\partial y_{j}}, \frac{\partial q_{l}}{\partial y_{l}} v\right) d t \\
\quad+\int_{0}^{T} 2 \operatorname{Im}\left(c_{j k} y_{k} \frac{\partial v}{\partial y_{j}}, q_{l} \frac{\partial v}{\partial y_{l}}\right) d t+\int_{0}^{T} 2 \operatorname{Im} \operatorname{tr}(C(t))\left(v, q_{l} \frac{\partial v}{\partial y_{l}}\right) d t \\
\quad-\int_{0}^{T} \operatorname{Im}\left(f, \frac{\partial q_{l}}{\partial y_{l}} v\right) d t-\int_{0}^{T} 2 \operatorname{Im}\left(f, q_{l} \frac{\partial v}{\partial y_{l}}\right) d t .
\end{aligned}
$$

Proof. Multiplying both sides of $(2.2)_{1}$, Section 2, by $q_{l}\left(\partial v / \partial y_{l}\right)$, integrating on $Q$, and taking the double of the imaginary parts of the resulting equality's both sides, we have

$$
\begin{gathered}
\int_{0}^{T} 2 \operatorname{Im}\left(v^{\prime}, q_{l} \frac{\partial v}{\partial y_{l}}\right) d t+\int_{0}^{T} 2 \operatorname{Re}\left(-\Delta v, q_{l} \frac{\partial v}{\partial y_{l}}\right) d t+\int_{0}^{T} 2 \operatorname{Im}\left(c_{j k} \cdot y_{k} \frac{\partial v}{\partial y_{j}}, q_{l} \frac{\partial v}{\partial y_{l}}\right) d t \\
+\int_{0}^{T} 2 \operatorname{Im} \operatorname{tr}(C(t))\left(v, q_{l} \frac{\partial v}{\partial y_{l}}\right) d t=\int_{0}^{T} 2 \operatorname{Im}\left(f, q_{l} \frac{\partial v}{\partial y_{l}}\right) d t .
\end{gathered}
$$

We have

$$
\frac{d}{d t}\left(v, q_{l} \frac{\partial v}{\partial y_{l}}\right)=\left(v^{\prime}, q_{l} \frac{\partial v}{\partial y_{l}}\right)+\left(v, q_{l} \frac{\partial v^{\prime}}{\partial y_{l}}\right)
$$


By Gauss' lemma, we obtain

$$
\int_{\Omega} \frac{\partial}{\partial y_{l}}\left(v q_{l} \bar{v}^{\prime}\right) d y=0, \quad \text { because } v=0 \text { on } \Sigma
$$

It follows that

$$
\left(v, q_{l} \frac{\partial v^{\prime}}{\partial y_{l}}\right)+\left(q_{l} \frac{\partial v}{\partial y_{l}}, v^{\prime}\right)+\left(\frac{\partial q_{l}}{\partial y_{l}} v, v^{\prime}\right)=0
$$

Substituting (3.5) in (3.3) and integrating on $(0, T)$, we obtain

$$
\int_{0}^{T}\left(v^{\prime}, q_{l} \frac{\partial v}{\partial y_{l}}\right) d t-\int_{0}^{T}\left(q_{l} \frac{\partial v}{\partial y_{l}}, v^{\prime}\right) d t=\left.\left(v, q_{l} \frac{\partial v}{\partial y_{l}}\right)\right|_{0} ^{T}+\int_{0}^{T}\left(\frac{\partial q_{l}}{\partial y_{l}} v, v^{\prime}\right) d t
$$

Since $z-\bar{z}=2 i \operatorname{Im} z$, the last identity implies

$$
2 \operatorname{Im} \int_{0}^{T}\left(v^{\prime}, q_{l} \frac{\partial v}{\partial y_{l}}\right) d t=-\left.i\left(v, q_{l} \frac{\partial v}{\partial y_{l}}\right)\right|_{0} ^{T}-i \int_{0}^{T}\left(\frac{\partial q_{l}}{\partial y_{l}} v, v^{\prime}\right) d t
$$

Taking the real parts of both sides of the above identity, we get

$$
2 \operatorname{Im} \int_{0}^{T}\left(v^{\prime}, q_{l} \frac{\partial v}{\partial y_{l}}\right) d t=\left.\operatorname{Im}\left(v, q_{l} \frac{\partial v}{\partial y_{l}}\right)\right|_{0} ^{T}-\operatorname{Im} \int_{0}^{T}\left(v^{\prime}, \frac{\partial q_{l}}{\partial y_{l}} v\right) d t
$$

(i) Analysis of $\operatorname{Im} \int_{0}^{T}\left(v^{\prime},\left(\partial q_{l} / \partial y_{l}\right) v\right) d t$.

Multiplying $(2.2)_{1}$ by $\left(\partial q_{l} / \partial y_{l}\right) v$, integrating on $\Omega$, and taking the imaginary parts of the resulting equality's both sides, we obtain

$$
\begin{gathered}
\operatorname{Im}\left(v^{\prime}, \frac{\partial q_{l}}{\partial y_{l}} v\right)+\operatorname{Re}\left(-\Delta v, \frac{\partial q_{l}}{\partial y_{l}} v\right)+\operatorname{Im}\left(c_{j k} \cdot y_{k} \frac{\partial v}{\partial y_{j}}, \frac{\partial q_{l}}{\partial y_{l}} v\right) \\
+\operatorname{Im}\left(\operatorname{tr}(C(t)) v, \frac{\partial q_{l}}{\partial y_{l}} v\right)=\operatorname{Im}\left(f, \frac{\partial q_{l}}{\partial y_{l}} v\right)
\end{gathered}
$$

By Green's formula, we obtain

$$
\operatorname{Re}\left(-\Delta v, \frac{\partial q_{l}}{\partial y_{l}} v\right)=\operatorname{Re}\left(\frac{\partial v}{\partial y_{j}}, \frac{\partial^{2} q_{l}}{\partial y_{j} \partial y_{l}} v\right)+\operatorname{Re}\left(\frac{\partial v}{\partial y_{j}}, \frac{\partial q_{l}}{\partial y_{l}} \frac{\partial v}{\partial y_{j}}\right)
$$

because $v=0$ on $\Gamma$. 
By (3.8), (3.9), and (3.10) we have

$$
\begin{aligned}
2 \operatorname{Im} & \int_{0}^{T}\left(v^{\prime}, q_{l} \frac{\partial v}{\partial y_{l}}\right) d t \\
= & \left.\operatorname{Im}\left(v, q_{l} \frac{\partial v}{\partial y_{l}}\right)\right|_{0} ^{T}-\int_{0}^{T} \operatorname{Im}\left(f, \frac{\partial q_{l}}{\partial y_{l}} v\right) d t+\int_{0}^{T} \operatorname{Re}\left(\frac{\partial v}{\partial y_{j}}, \frac{\partial^{2} q_{l}}{\partial y_{j} \partial y_{l}} v\right) d t \\
& +\int_{0}^{T}\left(\frac{\partial v}{\partial y_{j}}, \frac{\partial q_{l}}{\partial y_{l}} \frac{\partial v}{\partial y_{j}}\right) d t+\int_{0}^{T} \operatorname{Im}\left(c_{j k} \cdot y_{k} \frac{\partial v}{\partial y_{j}}, \frac{\partial q_{l}}{\partial y_{l}} v\right) d t
\end{aligned}
$$

(ii) Analysis of $\int_{0}^{T} 2 \operatorname{Re}\left(-\Delta v, q_{l}\left(\partial v / \partial y_{l}\right)\right) d t$.

By Gauss' lemma,

$$
\int_{\Omega} \frac{\partial}{\partial y_{j}}\left(\frac{\partial v}{\partial y_{j}} q_{l} \frac{\partial \bar{v}}{\partial y_{j}}\right) d y=\int_{\Gamma} q_{l} \cdot \nu_{l} \frac{\partial v}{\partial y_{j}} \frac{\partial \bar{v}}{\partial y_{j}} d \Gamma
$$

Since $\partial v / \partial y_{j}=v_{j}(\partial v / \partial v)$, we have from the last identity that

$$
2 \operatorname{Re}\left(\frac{\partial v}{\partial y_{j}}, q_{l} \frac{\partial}{\partial y_{j}}\left(\frac{\partial v}{\partial y_{j}}\right)\right)=-\left(\frac{\partial v}{\partial y_{j}}, \frac{\partial q_{l}}{\partial y_{j}} \frac{\partial v}{\partial y_{j}}\right)+\int_{\Gamma} q \cdot v\left|\frac{\partial v}{\partial v}\right|^{2} d \Gamma
$$

By Green's formula, we have

$$
\left(-\Delta v, q_{l} \frac{\partial v}{\partial y_{l}}\right)=\left(\frac{\partial v}{\partial y_{j}}, \frac{\partial q_{l}}{\partial y_{j}} \frac{\partial v}{\partial y_{l}}\right)+\left(\frac{\partial v}{\partial y_{j}}, q_{l} \frac{\partial}{\partial y_{j}}\left(\frac{\partial v}{\partial y_{j}}\right)\right)-\int_{\Gamma} q_{l} \cdot v_{l}\left|\frac{\partial v}{\partial \nu}\right|^{2} d \Gamma
$$

From (3.13), we modify (3.14) obtaining

$$
\begin{aligned}
& \int_{0}^{T} 2 \operatorname{Re}\left(-\Delta v, q_{l} \frac{\partial v}{\partial y_{l}}\right) d t \\
& \quad=\int_{0}^{T} 2 \operatorname{Re}\left(\frac{\partial v}{\partial y_{j}}, \frac{\partial q_{l}}{\partial y_{j}} \frac{\partial v}{\partial y_{l}}\right) d t-\int_{0}^{T} 2 \operatorname{Re}\left(\frac{\partial v}{\partial y_{j}}, \frac{\partial q_{l}}{\partial y_{l}} \frac{\partial v}{\partial y_{j}}\right) d t-\int_{0}^{T} \int_{\Gamma} q \cdot v\left|\frac{\partial v}{\partial v}\right|^{2} d \Gamma d t .
\end{aligned}
$$

Substituting (3.11) and (3.15) in the identity (3.2), we obtain the identity (3.1).

Before stating the direct inequality, the following consideration will be made. Let $\left(W,\|\cdot\|_{W}\right)$ be the Banach space of the weak solutions of (2.2), where $\|v\|_{W}=$ $\max _{0 \leq t \leq T}\|v(t)\|$. Considering by $\mathscr{F}=\left\{f \in L^{2}\left(0, T ; H_{0}^{1}(\Omega)\right) ; f^{\prime} \in L^{1}\left(0, T ; L^{2}(\Omega)\right)\right\}$ and denoting by $S$ the vector space of the strong solutions of $(2.2)$ relating to $\left\{v_{0}, f\right\} \in\left(H_{0}^{1}(\Omega) \cap\right.$ $\left.H^{2}(\Omega)\right) \times \mathscr{F}$, it follows that 
Lемма 3.2 (direct inequality). If $v$ is the weak solution of the boundary value problem (2.2), then there exists the inequality

$$
\int_{0}^{T} \int_{\Gamma}\left|\frac{\partial v}{\partial \nu}\right|^{2} d \Gamma d t \leq \alpha\left[\left\|v_{0}\right\|^{2}+\int_{0}^{T}\|f(s)\|^{2} d s\right]
$$

Proof. If $v$ is the strong solution of (2.2), it satisfies the identity of Lemma 3.1. If we choose the vector field $q=\left(q_{l}\right)_{1 \leq l \leq n}$, with $q \in\left[C^{2}(\bar{\Omega})\right]^{n}$, such that $q=v$ on $\Gamma$, where $v$ is the unit exterior normal vector to $\Gamma$, then $q \cdot v=1$ on $\Gamma$ and the left-hand side of the identity (3.1) is reduced to

$$
\int_{0}^{T} \int_{\Gamma}\left|\frac{\partial v}{\partial \nu}\right|^{2} d \Gamma d t
$$

The right-hand side of the identity (3.1), for $q=v$ on $\Gamma$, is bounded by

$$
\alpha\left(\max _{0 \leq t \leq T}\|v(t)\|^{2}+\int_{0}^{T}\|f(s)\|^{2} d s\right) .
$$

From the second estimate, we know that

$$
\|v\|^{2} \leq \alpha_{1}\left\|v_{0}\right\|^{2}
$$

Therefore, we obtain

$$
\int_{0}^{T} \int_{\Gamma}\left|\frac{\partial v}{\partial \nu}\right|^{2} d \Gamma d t \leq \alpha\left[\left\|v_{0}\right\|^{2}+\int_{0}^{T}\|f(s)\|^{2} d s\right] .
$$

Let us consider the operator $\gamma: S \rightarrow L^{2}(\Sigma)$ such that $\gamma(v)=\partial v / \partial v$ and the vector space $S$ is defined previously. From (3.1), we have

$$
|\gamma(v)|_{L^{2}(\Sigma)}^{2}=\int_{0}^{T} \int_{\Gamma}\left|\frac{\partial v}{\partial v}\right|^{2} d \Gamma d t \leq c \max _{0 \leq t \leq T}\|v(t)\|^{2}=c\|v\|_{W}^{2} .
$$

Thus, $\gamma$ is linear and continuous on $S$ that is dense in $W$. Therefore, $\gamma$ admits a linear and continuous extension to $\bar{S}=W$. Let $v$ be the weak solution relating to $v_{0}$ and $f$. Then, there exists a sequence of strong solutions $\left(v_{n}\right)$ such that $v_{n} \rightarrow v$ in $W$.

For each strong solution $v_{n}$, the inequality (3.21) is true, and therefore taking the limit we obtain

$$
\int_{0}^{T} \int_{\Gamma}\left|\frac{\partial v}{\partial \nu}\right|^{2} d \Gamma d t \leq \alpha\left[\left\|v_{0}\right\|^{2}+\int_{0}^{T}\|f(s)\|^{2} d s\right]
$$

now for the weak solution $v$.

The next inequality is true on a part $\Gamma\left(y_{0}\right)$ which we will define. In fact, let us consider a point $y_{0} \in \mathbb{R}^{n}$ and represent by $m(y)$ the vector $y-y_{0}$. We consider the following decomposition of $\Gamma$ :

$$
\Gamma\left(y_{0}\right)=\{y \in \Gamma ; C m(y) \cdot v \geq 0\}, \quad \Gamma_{*}\left(y_{0}\right)=\{y \in \Gamma ; C m(y) \cdot v<0\},
$$


where $C$ is the matrix $\left(K^{-1}(t)\right)^{\prime} K(t)$. We also define

$$
\sum\left(y_{0}\right)=\Gamma\left(y_{0}\right) \times(0, T), \quad \sum_{*}\left(y_{0}\right)=\Gamma_{*}\left(y_{0}\right) \times(0, T) .
$$

Lемма 3.3 (inverse inequality). If $v$ is a weak solution of the boundary value problem (2.2), with $f=0$, there exists the inequality

$$
\alpha\left\|v_{0}\right\|^{2} \leq \int_{0}^{T} \int_{\Gamma\left(y_{0}\right)}\left|\frac{\partial v}{\partial v}\right|^{2} d \Gamma d t
$$

where the constant $\alpha$ is independent of $v$ and depends only on $T,\left\|y_{0}\right\|$, and $\Omega$.

Proof. Let $v$ be the weak solution of (2.2) corresponding to $v_{0} \in H_{0}^{1}(\Omega)$. It follows from the identity (3.1), for $\vec{q}=C\left(y-y_{0}\right)$ and $f \equiv 0$, that

$$
\begin{aligned}
& \int_{0}^{T} \int_{\Gamma}(C m(y) \cdot v)\left|\frac{\partial v}{\partial \nu}\right|^{2} d \Gamma d t \\
& \quad=\left.(v, C m(y) \cdot \nabla v)\right|_{0} ^{T}+2 \int_{0}^{T} \operatorname{Re}(C \cdot \nabla v, \nabla v) d t+\int_{0}^{T} H(t) d t,
\end{aligned}
$$

where

$$
\begin{aligned}
H(t)= & \operatorname{Im}(C y \cdot \nabla v,(\operatorname{tr} C) v)+2 \operatorname{Im}(C y \cdot \nabla v, C m(y) \cdot \nabla v) \\
& +2 \operatorname{Im}((\operatorname{tr} C) v, C m(y) \cdot \nabla v) .
\end{aligned}
$$

We have

$$
\begin{aligned}
H(t)= & \operatorname{Im}((\operatorname{tr} C)(C y \cdot \nabla v), v)+2 \operatorname{Im}(C y \cdot \nabla v, C y \cdot \nabla v)-2 \operatorname{Im}\left(C y \cdot \nabla v, C y_{0} \cdot \nabla v\right) \\
& +2 \operatorname{Im}((\operatorname{tr} C) v, C y \cdot \nabla v)-2 \operatorname{Im}\left((\operatorname{tr} C) v, C y_{0} \cdot \nabla v\right) .
\end{aligned}
$$

Observe that if $z$ is a complex number $-2 \operatorname{Im} \bar{z}-\operatorname{Im} z=\operatorname{Im} z$, then

$$
2 \operatorname{Im}(v,(\operatorname{tr} C)(C y \cdot \nabla v))+\operatorname{Im}((\operatorname{tr} C)(C y \cdot \nabla v), v)=-\operatorname{Im}((\operatorname{tr} C)(C y \cdot \nabla v), v)
$$

Using (3.30) we modify $H$ obtaining

$$
\begin{aligned}
H(t)= & -\operatorname{Im}((\operatorname{tr} C)(C y \cdot \nabla v), v)+2 \operatorname{Im}|C y \cdot \nabla v|^{2}-2 \operatorname{Im}\left(C y \cdot \nabla v, C y_{0} \cdot \nabla v\right) \\
& -2 \operatorname{Im}\left((\operatorname{tr} C) v, C y_{0} \cdot \nabla v\right)=-\operatorname{Im}((\operatorname{tr} C)(C y \cdot \nabla v), v) \\
& -2 \operatorname{Im}\left(C y \cdot \nabla v, C y_{0} \cdot \nabla v\right)-2 \operatorname{Im}\left((\operatorname{tr} C) v, C y_{0} \cdot \nabla v\right) .
\end{aligned}
$$


Substituting (3.31) in (3.27) we find

$$
\begin{aligned}
& \int_{0}^{T} \int_{\Gamma}(C m(y) \cdot v)\left|\frac{\partial v}{\partial v}\right|^{2} d \Gamma d t \\
& =\left.(v, C m(y) \cdot \nabla v)\right|_{0} ^{T}+2 \int_{0}^{T} \operatorname{Re}(C \cdot \nabla v, \nabla v) d t-\int_{0}^{T} \operatorname{Im}(C y \cdot \nabla v, \operatorname{tr}(C) v) d t \\
& \quad-2 \int_{0}^{T} \operatorname{Im}\left(C y \cdot \nabla v, C y_{0} \cdot \nabla v\right) d t-2 \int_{0}^{T} \operatorname{Im}\left(\operatorname{tr}(C) v, C y_{0} \cdot \nabla v\right) d t
\end{aligned}
$$

Now we prove the inverse inequality (3.26). It will be done by steps.

Step 1. We prove that the weak solution of (2.2) satisfies

$$
\alpha_{1}\left|v_{0}\right|^{2}+\int_{0}^{T} \int_{\Gamma\left(y_{0}\right)}(C m(y) \cdot v)\left|\frac{\partial v}{\partial \nu}\right|^{2} d \Gamma d t \geq \alpha_{2}\left\|v_{0}\right\|^{2} .
$$

We note that from $(\mathrm{H} 2)$ we have

$$
2 \int_{0}^{T} \operatorname{Re}(C \cdot \nabla v, \nabla v) d t \geq 2 \alpha \int_{0}^{T}\|v\|^{2} d t
$$

Now, from the second estimate we have

$$
\begin{aligned}
\|v\|^{2}= & \left\|v_{0}\right\|^{2} \exp \left(-\int_{0}^{t} \operatorname{tr}(C) d r\right)-\operatorname{Im}\left(v_{0}, C y \cdot \nabla v_{0}\right) \exp \left(-\int_{0}^{t} \operatorname{tr}(C) d r\right) \\
& +\operatorname{Im}(v, C y \cdot \nabla v)-\int_{0}^{t} \operatorname{Im}\left(v, C^{\prime} y \cdot \nabla v\right) \exp \left(-\int_{0}^{t} \operatorname{tr}(C) d r\right) d s,
\end{aligned}
$$

therefore,

$$
\begin{aligned}
& 2 \int_{0}^{T} \operatorname{Re}(C \cdot \nabla v, \nabla v) d t \geq 2 \alpha \int_{0}^{T}\|v\|^{2} d t \\
& =2 \alpha\left\|v_{0}\right\|^{2} \int_{0}^{T} \exp \left(-\int_{0}^{t} \operatorname{tr}(C) d r\right) d t-2 \alpha \int_{0}^{T} \operatorname{Im}\left(v_{0}, C y \cdot \nabla v_{0}\right) \exp \left(-\int_{0}^{t} \operatorname{tr}(C) d r\right) d t \\
& \quad+2 \alpha \int_{0}^{T} \operatorname{Im}(v, C y \cdot \nabla v) d t-2 \alpha \int_{0}^{T} \int_{0}^{t} \operatorname{Im}\left(v, C^{\prime} y \cdot \nabla v\right) \exp \left(-\int_{0}^{t} \operatorname{tr}(C) d r\right) d s d t .
\end{aligned}
$$

Observe that for each $e_{j k}=\left(\delta_{j}^{k}\right), k=1, \ldots, n$, where

$$
\delta_{j}^{k}= \begin{cases}1 & \text { if } j=k \\ 0 & \text { if } j \neq k\end{cases}
$$

we have, from (H2), that $C(t) e_{j k} \cdot e_{j k} \geq \alpha\left|e_{j k}\right|^{2}=\alpha$ and $C(t) e_{j k} \cdot e_{j k}=C_{k k}(t)$, for all $k=$ $1, \ldots, n$ for all $t \in[0, T]$, then $C_{k k}(t) \geq \alpha>0$ and, therefore,

$$
\operatorname{tr}(C) \geq n \alpha>0
$$


From (3.38), it follows that, for all $s, t \in[0, T], s \leq t$,

$$
0 \leq \int_{s}^{t} \operatorname{tr}(C) d r \leq \int_{0}^{T} \operatorname{tr}(C) d r
$$

and then

$$
\exp \left(-\int_{0}^{T} \operatorname{tr}(C) d r\right) \leq \exp \left(-\int_{s}^{t} \operatorname{tr}(C) d r\right) \leq 1
$$

Therefore,

$$
\begin{aligned}
2 \alpha\left\|v_{0}\right\|^{2} \int_{0}^{T} \exp \left(-\int_{0}^{t} \operatorname{tr}(C) d r\right) d t \\
\quad \geq 2 \alpha\left\|v_{0}\right\|^{2} \int_{0}^{T} \exp \left(-\int_{0}^{T} \operatorname{tr}(C) d r\right) d t \geq 2 \alpha T C_{1}(T)\left\|v_{0}\right\|^{2} .
\end{aligned}
$$

We also have

$$
\begin{gathered}
-2 \alpha \int_{0}^{T} \operatorname{Im}\left(v_{0}, C y \cdot \nabla v_{0}\right) \exp \left(-\int_{0}^{t} \operatorname{tr}(C) d r\right) d t \\
\geq-2 \alpha\left|v_{0}\right||| v_{0}|| \int_{0}^{T}|C(t) y| d t .
\end{gathered}
$$

Observe that $|C(t) y|=|y|$ for all $t \in[0, T]$, because $\{C(t)\}_{t \geq 0}$ is a family of orthogonal matrices of $\mathbb{R}^{n}$. Therefore,

$$
\int_{0}^{T}|C(t) y| d t \leq M(T, \Omega)
$$

Substituting (3.43) in (3.42) we obtain

$$
-2 \alpha \int_{0}^{T} \operatorname{Im}\left(v_{0}, C y \cdot \nabla v_{0}\right) \exp \left(-\int_{0}^{t} \operatorname{tr}(C) d r\right) d t \geq-2 \alpha M(T, \Omega)\left|v_{0}\right|\left\|v_{0}\right\|
$$

From Young's inequality we get

$$
-2 \alpha \int_{0}^{T} \operatorname{Im}\left(v_{0}, C y \cdot \nabla v_{0}\right) \exp \left(-\int_{0}^{t} \operatorname{tr}(C) d r\right) d t \geq \frac{-(\alpha M(T, \Omega))^{2}}{\epsilon}\left|v_{0}\right|^{2}-\epsilon\left\|v_{0}\right\|^{2},
$$

where $\epsilon>0$.

(i) Analysis of $2 \alpha \int_{0}^{T} \operatorname{Im}(v, C y \cdot \nabla v) d t$.

We observe that from the second estimate there exists a constant $\alpha_{1}>0$ such that

$$
\|v(t)\| \leq K_{1}\left\|v_{0}\right\|
$$


18 Schrödinger equations in noncylindrical domains

Therefore, from Young's inequality, it follows that

$$
\begin{aligned}
2 \alpha \int_{0}^{T} \operatorname{Im}(v, C y \cdot \nabla v) d t & \geq-2 \alpha_{2} \int_{0}^{T}\left|v_{0}\right||C y|\left\|v_{0}\right\| d t \\
& \geq \frac{-M(\Omega, T)^{2}}{\epsilon}\left|v_{0}\right|^{2}-\epsilon\left\|v_{0}\right\|^{2}
\end{aligned}
$$

(ii) Analysis of $-2 \alpha \int_{0}^{T} \int_{0}^{t} \operatorname{Im}\left(v, C^{\prime} y \cdot \nabla v\right) \exp \left(-\int_{0}^{t} \operatorname{tr}(C) d r\right) d s d t$.

$$
\begin{aligned}
& -2 \alpha \int_{0}^{T} \int_{0}^{t} \operatorname{Im}\left(v, C^{\prime} y \cdot \nabla v\right) \exp \left(-\int_{0}^{t} \operatorname{tr}(C) d r\right) d s d t \\
& \quad \geq-2 \alpha \int_{0}^{T} \int_{0}^{t}|v|\left|C^{\prime} y\right|\|v\| d s d t \geq-2 \alpha_{3} \int_{0}^{T} \int_{0}^{t}\left|v_{0}\right|\left|C^{\prime} y\right| \| v_{0}|| d s d t \\
& \quad \geq \frac{(M(\Omega, T, n))}{\epsilon}\left|v_{0}\right|^{2}-\epsilon\left\|v_{0}\right\|^{2} .
\end{aligned}
$$

Substituting (3.42), (3.45), (3.47), and (3.48) in (3.36) we obtain

$$
2 \int_{0}^{T} \operatorname{Re}(C \cdot \nabla v, \nabla v) d t \geq(2 M(\alpha, T)-3 \epsilon)\left\|v_{0}\right\|^{2}-\frac{(M(\alpha, T, n, \Omega))^{2}}{\epsilon}\left|v_{0}\right|^{2}
$$

Choosing $\epsilon>0$ such that $0<\epsilon<2 M(\alpha, T) / 3$, then we have

$$
2 \int_{0}^{T} \operatorname{Re}(C \cdot \nabla v, \nabla v) d t \geq \alpha_{4}(T)|| v_{0} \|^{2}-\alpha_{5}(T)\left|v_{0}\right|^{2},
$$

where $\alpha_{4}(T)$ and $\alpha_{5}(T)$ are positive constants.

(iii) Analysis of $-2 \int_{0}^{T} \operatorname{Im}\left(C y \cdot \nabla v, C y_{0} \cdot \nabla v\right) d t$.

$$
\begin{aligned}
-2 \int_{0}^{T} \operatorname{Im}\left(C y \cdot \nabla v, C y_{0} \cdot \nabla v\right) d t & \geq-2 \int_{0}^{T}|C y|\|v\|\left|C y_{0}\right|\|v\| d t \\
& \geq-2 M(\Omega) \int_{0}^{T}\|v\|^{2} d t .
\end{aligned}
$$

From (3.35) and (3.40), it follows that

$$
\begin{aligned}
\int_{0}^{T}\|v\|^{2} d t \leq & \left\|v_{0}\right\|^{2} T+\int_{0}^{T}\left|\left(v_{0}, C y \cdot \nabla v_{0}\right)\right| d t \\
& +\int_{0}^{T}|(v, C y \cdot \nabla v)| d t+T \int_{0}^{T}\left|\left(v, C^{\prime} y \cdot \nabla v\right)\right| d t \\
\leq & \left\|v_{0}\right\|^{2} T \leq \frac{M(\Omega, T, n)}{2 \epsilon}\left|v_{0}\right|^{2}+\frac{3 \epsilon}{2}\left\|v_{0}\right\|^{2} .
\end{aligned}
$$

Substituting (3.52) in (3.51), we get

$$
\begin{aligned}
& -2 \int_{0}^{T} \operatorname{Im}\left(C y \cdot \nabla v, C y_{0} \cdot \nabla v\right) d t \\
& \quad \geq-2 M(\Omega, T)\left\|v_{0}\right\|^{2}-\frac{M(\Omega, T, n)}{\epsilon}\left|v_{0}\right|^{2}-\epsilon M(\Omega)\left\|v_{0}\right\|^{2}
\end{aligned}
$$


(iv) Analysis of $-2 \int_{0}^{T} \operatorname{Im}\left(\operatorname{tr}(C) v, C y_{0} \cdot \nabla v\right) d t$.

$$
\begin{aligned}
-2 \int_{0}^{T} \operatorname{Im}\left(\operatorname{tr}(C) v, C y_{0} \cdot \nabla v\right) d t & \geq-2 \int_{0}^{T}|\operatorname{tr}(C) v|\left|C y_{0}\right||\nabla v| d t \\
& \geq-\frac{(M(T, \Omega))^{2}}{\epsilon}\left|v_{0}\right|^{2}-\epsilon\left\|v_{0}\right\|^{2}
\end{aligned}
$$

(v) Analysis of $-\int_{0}^{T} \operatorname{Im}(C y \cdot \nabla v, \operatorname{tr}(C) v) d t$.

$$
\begin{aligned}
& -\int_{0}^{T} \operatorname{Im}(C y \cdot \nabla v,(\operatorname{tr} C) v) d t \\
& \quad \geq-\int_{0}^{T}|C y|\|v\||(\operatorname{tr} C) v| d t \geq-\int_{0}^{T} M(\Omega, T)|| v(t)|||v(t)| d t \\
& \quad \geq-M\left(\Omega, T, \alpha_{1}\right)|| v_{0}||\left|v_{0}\right| \geq-\frac{\left(M\left(\Omega, T, \alpha_{1}\right)\right)^{2}}{2 \epsilon}\left|v_{0}\right|^{2}-\frac{\epsilon}{2}\left\|v_{0}\right\|^{2} .
\end{aligned}
$$

We also have

$$
\left.(v, C m(y) \cdot \nabla v)\right|_{0} ^{T}=(v(T), C m(y) \cdot \nabla v(T))-\left(v_{0}, C m(y) \cdot \nabla v_{0}\right),
$$

therefore, from Young's inequality, it follows that

$$
\left.(v, C m(y) \cdot \nabla v)\right|_{0} ^{T} \geq \frac{\left(M\left(\Omega, \alpha_{1}\right)\right)^{2}}{2 \epsilon}\left|v_{0}\right|^{2}-\frac{\epsilon}{2}\left\|v_{0}\right\|^{2} .
$$

Substituting (3.50), (3.53), (3.54), (3.55), and (3.57) in (3.32) we obtain, after some computations, that the weak solution of (2.2) satisfies (3.33).

Step 2. We prove in this step that if $v$ is a weak solution of (2.2) then there exists a constant $\lambda>0$ such that

$$
\lambda\left|v_{0}\right|^{2} \leq \int_{0}^{T} \int_{\Gamma\left(y_{0}\right)}(C m(y) \cdot v)\left|\frac{\partial v}{\partial \nu}\right|^{2} d \Gamma d t .
$$

In fact, we argue by contradiction. Suppose (3.58) is false. Considering $v_{0} \in H_{0}^{1}(\Omega)$, there exists a sequence $\left(v_{\mu}\right)$ of strong solutions of $(2.2), v_{\mu}(0)=v_{0 \mu}$, such that

$$
\int_{0}^{T} \int_{\Gamma\left(y_{0}\right)}(C m(y) \cdot v)\left|\frac{\partial v}{\partial \nu}\right|^{2} d \Gamma d t \leq \frac{1}{\mu}\left|v_{0 \mu}\right|^{2},
$$

and we can suppose $\left|v_{0 \mu}\right|=1$. Then, from (3.59), we obtain

$$
\lim _{\mu \rightarrow \infty} \int_{0}^{T} \int_{\Gamma\left(y_{0}\right)}(C m(y) \cdot v)\left|\frac{\partial v}{\partial \nu}\right|^{2} d \Gamma d t=0
$$

strongly. 
In Step 1 we proved that

$$
C_{1}\left|v_{0 \mu}\right|^{2}+\int_{0}^{T} \int_{\Gamma\left(y_{0}\right)}(C m(y) \cdot v)\left|\frac{\partial v_{\mu}}{\partial \nu}\right|^{2} d \Gamma d t \geq C_{2}\left\|v_{0 \mu}\right\|^{2}
$$

The left-hand side of (3.61) is bounded, then it follows that $\left(v_{0 \mu}\right)$ is bounded in $H_{0}^{1}(\Omega)$. Since the embedding of $H_{0}^{1}(\Omega)$ in $L^{2}(\Omega)$ is compact, it follows that we can extract a subsequence, still represented by $\left(v_{0 \mu}\right)$, such that

$$
\lim _{\mu \rightarrow \infty} v_{0 \mu}=v_{0} \quad \text { strongly in } L^{2}(\Omega) .
$$

Since $\left|v_{0 \mu}\right|=1$, it follows that

$$
\left|v_{0}\right|=1
$$

We also have

$$
\left|v_{\mu}(t)-v_{\eta}(t)\right| \leq C(T)\left|v_{0 \mu}-v_{0 \eta}\right|
$$

which implies that

$$
\lim _{\mu \rightarrow \infty} v_{\mu}=v \quad \text { in } C^{0}\left([0, T] ; L^{2}(\Omega)\right) .
$$

Integrating by parts for $\xi \in L^{\infty}\left(0, T ; H_{0}^{1}(\Omega) \cap H^{2}(\Omega)\right)$ and $\xi^{\prime} \in L^{\infty}\left(0, T ; L^{2}(\Omega)\right)$, with $\xi(0)=\xi(T)=0$, we obtain

$$
\int_{Q} v_{\mu}\left(-\overline{\xi^{\prime}}-i \Delta \bar{\xi}-(C y \cdot \nabla \bar{\xi})\right) d y d t=0
$$

When $\mu \rightarrow \infty$ in (3.66) we have

$$
\mid \begin{aligned}
& \int_{Q} v\left(\overline{\xi^{\prime}}+i \Delta \bar{\xi}+(C y \cdot \nabla \bar{\xi})\right) d y d t=0 \\
& v(0 g)=v_{0}
\end{aligned}
$$

We transform (3.67) into a noncylindrical problem on $\hat{Q}$ by the mapping $y=K^{-1}(t) x$, $x \in \widehat{Q}$. Consider $\theta(x, t)=v\left(K^{-1}(t) x, t\right)$, where $v$ is the weak solution, then $\theta \in C^{0}([0, T]$; $\left.H_{0}^{1}(\Omega)\right)$.

Represent by $\hat{G}$ a bounded convex set of $\mathbb{R}^{n}$ such that its closure contains $\hat{Q}$ and $\hat{\Sigma}\left(y_{0}\right)$. Let $O$ be $\hat{G} \cap \widehat{Q} \neq \varnothing$ and let $\tilde{\theta}(x, t)$ equal to $\theta(x, t)$ on $\hat{Q}$ and zero outside. We prove that

$$
\tilde{\theta}^{\prime}-i \Delta \tilde{\theta}=0 \quad \text { in the sense of } \mathscr{D}^{\prime}(\hat{G}) \text {. }
$$

By definition, $\tilde{\theta}=0$ on $\hat{G} \backslash \hat{Q}$, then $\tilde{\theta}=0$ on $\hat{G}$ by Holmgren's theorem. Therefore, $\theta=$ $\tilde{\theta}=0$ on $\hat{Q}$ and then $v=0$. This is a contradiction because $\left|v_{0}\right|=1$. 


\section{Ultra weak solutions or solutions by transposition}

Let us consider the following nonhomogeneous mixed problem:

$$
\mid \begin{aligned}
& L v=0 \quad \text { on } Q \\
& v=w \quad \text { on } \Sigma \\
& v(0)=v_{0} \quad \text { in } \Omega
\end{aligned}
$$

where $L v=v^{\prime}-i \Delta v+C y \cdot \nabla v$ was defined in Section 2.

We are looking for a concept of a solution for (4.1). Let us suppose $w \in L^{2}(\Sigma), v_{0} \in$ $H^{-1}(\Omega)$ and consider a function $\theta=\theta(y, t)$ such that $\theta=0$ on $\Sigma$ and $\theta(T)=\theta(y, T)=0$ for $y \in \Omega$. Multiplying both sides of (4.1) 1 by $\bar{\theta}$ and integrating on $Q$, we obtain

$$
\int_{0}^{T} \int_{\Omega}\left(v^{\prime}-i \Delta v+C y \cdot \nabla v\right) \bar{\theta} d y d t=0
$$

We have the following.

(i)

$$
\int_{0}^{T} \int_{\Omega} v^{\prime} \bar{\theta} d y d t=\int_{0}^{T}\left(v^{\prime}, \theta\right) d t=(v(T), \theta(T))-(v(0), \theta(0))-\int_{0}^{T}\left(v, \theta^{\prime}\right) d t
$$

or

$$
\int_{0}^{T} \int_{\Omega} v^{\prime} \bar{\theta} d y d t=-(v(0), \theta(0))-\int_{0}^{T} \int_{\Omega} v \overline{\theta^{\prime}} d y d t
$$

(ii) $-\int_{0}^{T} \int_{\Omega} i \Delta v \bar{\theta} d y d t$

By Green's formula,

$$
\begin{aligned}
& -\int_{\Omega} \Delta v \bar{\theta} d y=\int_{\Omega} \nabla v \nabla \bar{\theta} d y-\int_{\Gamma} \frac{\partial v}{\partial \nu} \bar{\theta} d \Gamma \\
& -\int_{\Omega} v \Delta \bar{\theta} d y=\int_{\Omega} \nabla v \nabla \bar{\theta} d y-\int_{\Gamma} v \frac{\partial \bar{\theta}}{\partial \nu} d \Gamma .
\end{aligned}
$$

Observe that by hypothesis $\theta=0$ on $\Sigma$. Then,

$$
-i \int_{0}^{T} \int_{\Omega} \Delta v \bar{\theta} d y d t=-i \int_{0}^{T} \int_{\Omega} v \Delta \bar{\theta} d y d t+i \int_{0}^{T} \int_{\Gamma} v \frac{\partial \bar{\theta}}{\partial \nu} d \Gamma d t
$$

(iii) $\int_{0}^{T} \int_{\Omega} C y \cdot \nabla v \bar{\theta} d y d t$.

By Gauss' lemma we have

$$
\int_{\Omega} \frac{\partial}{\partial y_{l}}\left(v_{l k} y_{k} \bar{\theta}\right) d y=0,
$$

because $\theta=0$ on $\Sigma$. 
From (4.7), it follows that

$$
\int_{\Omega} \frac{\partial v}{\partial y_{l}} c_{l k} y_{k} \bar{\theta} d y+\int_{\Omega} v c_{l k} \delta_{l}^{k} \bar{\theta} d y+\int_{\Omega} v c_{l k} y_{k} \frac{\partial \bar{\theta}}{\partial y_{l}} d y=0
$$

or

$$
\int_{0}^{T} \int_{\Omega} C y \cdot \nabla v \bar{\theta} d y d t=-\int_{0}^{T} \int_{\Omega} \operatorname{tr}(C(t)) v \bar{\theta} d y d t-\int_{0}^{T} \int_{\Omega} v C y \cdot \nabla \bar{\theta} d y d t .
$$

Adding (4.4), (4.6), and (4.9) and observing (4.2), we get

$$
-\left(v_{0}, \theta(0)\right)+\int_{0}^{T} \int_{\Gamma} v \frac{\partial \bar{\theta}}{\partial \nu} d \Gamma d t+\int_{Q} v\left(-\overline{\theta^{\prime}}-i \Delta \bar{\theta}-\operatorname{tr}(C(t)) \bar{\theta}-C y \cdot \nabla \bar{\theta}\right) d y d t=0
$$

Hence,

$$
-\int_{Q} v \overline{\left(\theta^{\prime}-i \Delta \theta+\operatorname{tr}(C) \theta+C y \cdot \nabla \theta\right)} d y d t=\left(v_{0}, \theta(0)\right)-i \int_{0}^{T} \int_{\Gamma} v \frac{\partial \bar{\theta}}{\partial \nu} d \Gamma d t
$$

Now, we formulate the concept of solution by transposition or ultra weak solution for the problem (4.1).

Given $f \in L^{1}\left(0, T ; H_{0}^{1}(\Omega)\right)$, let $\theta$ be the weak solution of the following backward mixed problem

$$
\mid \begin{aligned}
& L^{*} \theta=-f \quad \text { in } Q, \\
& \theta=0 \quad \text { on } \Sigma \\
& \theta(T)=0 \quad \text { on } \Omega,
\end{aligned}
$$

where $L^{*} \theta=\theta^{\prime}-i \Delta \theta+\operatorname{tr}(C) \theta+C y \cdot \nabla \theta$ according to Section 2 .

Reversing time in (4.12) and defining $\hat{\theta}(y, t)=\theta(y, T-t), \hat{f}(y, t)=f(y, T-t)$, and $\widehat{C}(t)=-C(T-t)$, we obtain

$$
\hat{\theta}^{\prime}+i \Delta \hat{\theta}+\operatorname{tr}(\hat{C}) \hat{\theta}+\hat{C} y \cdot \nabla \hat{\theta}=\hat{f}
$$

Then, we obtain from (4.12) the equivalent problem

$$
\mid \begin{aligned}
& \overline{\hat{\theta}^{\prime}}-i \Delta \overline{\hat{\theta}}+\operatorname{tr}(\hat{C}) \overline{\hat{\theta}}+\hat{C} y \cdot \nabla \overline{\hat{\theta}}=\overline{\hat{f}} \quad \text { in } Q, \\
& \overline{\hat{\theta}}=0 \quad \text { on } \Sigma, \\
& \overline{\hat{\theta}}(0)=0 \quad \text { on } \Omega,
\end{aligned}
$$

which, by Section 2, Theorem 2.1, has only one weak solution $\overline{\hat{\theta}}$. Therefore, we have the following definition. 
Definition 4.1. For $v_{0} \in H^{-1}(\Omega)$ and $w \in L^{2}(\Sigma)$, the solution by transposition of the problem $(4.1)$ is called the unique function $v \in L^{\infty}\left(0, T ; H^{-1}(\Omega)\right)$ such that

$$
\int_{0}^{T}\langle v(t), f(t)\rangle_{H^{-1}(\Omega) \times H_{0}^{1}(\Omega)} d t=\left\langle v_{0}, \theta(0)\right\rangle-\int_{\Sigma} w i \frac{\partial \bar{\theta}}{\partial \nu} d \Gamma d t
$$

for all $f \in L^{1}\left(0, T ; H_{0}^{1}(\Omega)\right)$, where $\theta$ is the weak solution of (4.12).

Theorem 4.2. Given $v_{0} \in H^{-1}(\Omega)$ and $w \in L^{2}(\Sigma)$, there exists only one ultra weak solution $v \in L^{\infty}\left(0, T ; H^{-1}(\Omega)\right)$ of the nonhomogeneous boundary value problem (4.1).

Proof. The existence is a consequence of Riesz representation theorem for continuous linear functional on $L^{1}\left(0, T ; H_{0}^{1}(\Omega)\right)$.

For the uniqueness, suppose we have two ultra weak solutions $v$ and $\hat{v}$ corresponding to $v_{0}$ and $f$. Then, by definition of ultra weak solution, we have

$$
\langle v-\hat{v}, f\rangle=0
$$

where $\langle\cdot, \cdot\rangle$ is the duality between $L^{\infty}\left(0, T ; H^{-1}(\Omega)\right)$ and $L^{1}\left(0, T ; H_{0}^{1}(\Omega)\right)$. Then, by HahnBanach theorem, we have $v-\hat{v}=0$.

\section{Exact controllability}

In this section, at first, we solve the problem of the exact controllability for the nonhomogeneous boundary value problem on the cylinder $Q$ :

$$
\mid \begin{aligned}
& v^{\prime}-i \Delta v+C y \cdot \nabla v=0 \quad \text { on } Q \\
& v=w \quad \text { on } \Sigma \\
& v(0)=v_{0} \quad \text { in } \Omega .
\end{aligned}
$$

Later, we obtain the exact controllability result for the nonhomogeneous mixed problem (1.5) on the noncylindrical domain $\hat{Q}$.

The problem of exact controllability for (5.1) can be formulated as follows: given $T>$ 0 , find a Hilbert space $H$ such that for each initial data $v_{0} \in H$, there exists a control $w$ belonging to a space of controls on $\Sigma$ such that the corresponding solution $v(y, t, w)$ of (5.1) verifies the condition

$$
v(y, T, w)=0 \quad \forall y \in \Omega
$$

TheOREM 5.1. Let $\Omega$ be a regular, bounded open set of $\mathbb{R}^{n}$ and suppose that $(\mathrm{H} 1)$ and $(\mathrm{H} 2)$ hold. If $T>0$, then, for each $v_{0} \in H^{-1}(\Omega)$, there exists a control $w \in L^{2}(\Sigma)$ such that $v$, solution of problem (5.1), satisfies the condition

$$
v(y, T, w)=0 \quad \forall y \in \Omega .
$$

Proof. In order to prove the exact controllability for (5.1), we employ HUM (hilbert uniqueness method) idealized by Lions [8]. We describe the method by steps. 
24 Schrödinger equations in noncylindrical domains

Step 1. Given $\phi_{0} \in \mathscr{D}(\Omega)$, let us consider the adjoint problem

$$
\mid \begin{aligned}
& \phi^{\prime}-i \Delta \phi+C y \cdot \nabla \phi+\operatorname{tr}(C) \phi=0 \quad \text { in } Q, \\
& \phi=0 \quad \text { on } \Sigma, \\
& \phi(0)=\phi_{0} \quad \text { in } \Omega .
\end{aligned}
$$

We know by Section 1 that (5.4) has a strong solution. By the direct inequality, we obtain

$$
\frac{\partial \phi}{\partial v} \in L^{2}(\Sigma)
$$

Step 2. We solve the nonhomogeneous backward problem

$$
\mid \begin{aligned}
& \psi^{\prime}-i \Delta \psi+C y \cdot \nabla \psi=0 \quad \text { in } Q \\
& \psi= \begin{cases}-i \frac{\partial \phi}{\partial \nu} & \text { on } \Sigma\left(y_{0}\right), \\
0 & \text { in } \Sigma \backslash \Sigma\left(y_{0}\right),\end{cases} \\
& \psi(T)=0 .
\end{aligned}
$$

Note that (5.6) is a nonhomogeneous backward problem of the type studied in Section 4. To obtain, from (5.6), the system (4.1) of Section 4, it is sufficient to consider the change of variable $T-t$ in place of $t$.

The operator $\Lambda$. From the solution $\psi$ of (5.6), we define the application

$$
\Lambda\left\{\phi_{0}\right\}=\psi(0)
$$

Observe that, from $\phi_{0} \in \mathscr{D}(\Omega)$, we obtain the solution $\phi$ of (5.4) with regularity (5.5) for the normal derivative. Then, the problem (5.6) is well posed, from which we define $\Lambda$. Step 3. Multiplying both sides of (5.6) by $\phi$, the solution of (5.4), and integrating on $Q$ we obtain

$$
0=\langle L \psi, \phi\rangle=\left\langle\psi, L^{*} \phi\right\rangle+\langle\psi(T), \phi(T)\rangle-\langle\psi(0), \phi(0)\rangle+i \int_{0}^{T} \int_{\Gamma} \psi \frac{\partial \bar{\phi}}{\partial \nu} d \Gamma d t .
$$

That is,

$$
0=-\left\langle\psi(0), \phi_{0}\right\rangle+\int_{0}^{T} \int_{\Gamma}\left|\frac{\partial \phi}{\partial \nu}\right|^{2} d \Gamma d t
$$

Therefore,

$$
\left\langle\psi(0), \phi_{0}\right\rangle=\int_{0}^{T} \int_{\Gamma}\left|\frac{\partial \phi}{\partial \nu}\right|^{2} d \Gamma d t
$$

Substituting (5.7) in (5.10), we obtain

$$
\left\langle\Lambda\left\{\phi_{0}\right\}, \phi_{0}\right\rangle=\int_{0}^{T} \int_{\Gamma}\left|\frac{\partial \phi}{\partial v}\right|^{2} d \Gamma d t
$$


If we consider $\phi_{0}, \zeta_{0} \in \mathscr{D}(\Omega)$ and we represent by $\phi$ and $\zeta$ the corresponding weak solution of (5.4), we obtain, by the same argument used to obtain (5.10), that

$$
\left\langle\Lambda\left\{\phi_{0}\right\}, \zeta_{0}\right\rangle=\int_{0}^{T} \int_{\Gamma} \frac{\partial \phi}{\partial \nu} \frac{\partial \bar{\zeta}}{\partial \nu} d \Gamma d t
$$

We define in $\mathscr{D}(\Omega)$ the quadratic form

$$
\left\|\phi_{0}\right\|_{F}^{2}=\int_{0}^{T} \int_{\Gamma}\left|\frac{\partial \phi}{\partial \nu}\right|^{2} d \Gamma d t
$$

Remark 5.2. It follows from the direct inequality that the quadratic form defined above is a norm in $\mathscr{D}(\Omega)$, induced by the inner product

$$
(\phi, \zeta)_{F}=\int_{0}^{T} \int_{\Gamma} \frac{\partial \phi}{\partial \nu} \frac{\partial \bar{\zeta}}{\partial \nu} d \Gamma d t
$$

defined in $\mathscr{D}(\Omega) \times \mathscr{D}(\Omega)$.

Let us represent by $F$ the completion of $\mathscr{D}(\Omega)$ with the Hilbertian norm (5.13).

It follows from the remark above and (5.11) that

$$
\left\langle\Lambda\left\{\phi_{0}\right\}, \zeta_{0}\right\rangle=\left(\phi_{0}, \zeta_{0}\right)
$$

Then, $b\left(\phi_{0}, \zeta_{0}\right)=\left\langle\Lambda\left\{\phi_{0}\right\}, \zeta_{0}\right\rangle$ is a sesquilinear form, Hermitian, and strictly positive in $\mathscr{D}(\Omega) \times \mathscr{D}(\Omega)$. Its continuity follows from Schwarz inequality. In fact,

$$
\left|b\left(\phi_{0}, \zeta_{0}\right)\right|=\left|\left\langle\Lambda\left\{\phi_{0}\right\}, \zeta_{0}\right\rangle\right| \leq\left\|\phi_{0}\right\|_{F}\left\|\zeta_{0}\right\|_{F}
$$

It follows that $b\left(\phi_{0}, \zeta_{0}\right)$ has a unique extension by closure, to the completion $F$ of $\mathscr{D}(\Omega)$. Let us still represent by $b\left(\phi_{0}, \zeta_{0}\right)$ this extension. It is sesquilinear, Hermitian, and strictly positive in $F$. Then, by Lax-Milgram's lemma, given $v_{0} \in F^{\prime}$, dual of $F$, there exists a unique $\phi_{0} \in F$ such that

$$
\left\langle\Lambda\left\{\phi_{0}\right\}, \zeta_{0}\right\rangle=b\left(\phi_{0}, \zeta_{0}\right)=\left\langle v_{0}, \zeta_{0}\right\rangle \quad \forall \zeta_{0} \in F
$$

This means that, given $v_{0} \in F^{\prime}$, there exists a unique $\phi_{0} \in F$ such that

$$
\Lambda\left\{\phi_{0}\right\}=v_{0} \quad \text { in } F^{\prime}
$$

But, by (5.7), we have $\Lambda\left\{\phi_{0}\right\}=\psi(0)$, which implies that the ultra weak solution $\psi$ of the backward problem (5.6) satisfies the initial condition $\psi(0)=v_{0}$. It follows, by uniqueness, that $\psi=v$ and then $v(T)=0$, which is the condition (5.2).

We need only to characterize, in terms of space of functions on $\Omega$, the completion $F$ of $\mathscr{D}(\Omega)$ with respect to the norm $\|\cdot\|_{F}$ given by (5.13).

In fact, from inverse and direct inequalities, we have

$$
C_{0}\left\|\phi_{0}\right\|^{2} \leq \int_{0}^{T} \int_{\Gamma}\left|\frac{\partial \phi}{\partial \nu}\right|^{2} d \Gamma d t \leq C_{1}\left\|\phi_{0}\right\|^{2},
$$


which implies that the norm $\|\cdot\|_{F}$ in $\mathscr{D}(\Omega)$, defined in (5.13), is equivalent to the norm $H_{0}^{1}(\Omega)$. Therefore, $F=H_{0}^{1}(\Omega)$ and $F^{\prime}=H^{-1}(\Omega)$.

The proof of Theorem 1.1 follows from the previous theorem and the diffeomorphism $\tau$ defined in Section 1 .

\section{Appendix}

The objective of this appendix is to prove an identity in order to modify the surface integral

$$
\int_{\Gamma} v_{j} \cdot c_{j k}(t) y_{k}\left|\frac{\partial v_{m}}{\partial \nu}\right|^{2} d \Gamma
$$

that appears in the second estimate.

In fact, let us consider the approximate equation

$$
\left(v_{m}^{\prime}(t), w_{j}\right)+i\left(\left(v_{m}(t), w_{j}\right)\right)+\left(C(t) y \cdot \nabla v_{m}(t), w_{j}\right)+\operatorname{tr}(C(t))\left(v_{m}(t), w_{j}\right)=\left(f(t), w_{j}\right)
$$

Let us consider $L^{2}(\Omega)=V_{m} \oplus V_{m}^{\perp}$ and $P_{m}$ the orthogonal projection from $L^{2}(\Omega)$ on $V_{m}$. We know that

(i) $P_{m}$ is bounded, self-adjoint;

(ii) $P_{m} w=w$ for all $w \in V_{m}$;

(iii) $P_{m} w=\sum_{j=1}^{m}\left(w, w_{j}\right) w_{j}$ for each $w \in L^{2}(\Omega)$.

Multiplying both sides of the approximate equation (A.2) by $w_{j}$ and adding for $1 \leq$ $j \leq m$, we obtain that

$$
v_{m}^{\prime}-i \Delta v_{m}+P_{m}\left[C(t) y \cdot \nabla v_{m}\right]+\operatorname{tr}(C(t)) v_{m}=P_{m} f
$$

Taking the inner product of both sides of (A.3) with $C(t) y \cdot \nabla v_{m}$, we obtain

$$
\begin{gathered}
\left(v_{m}^{\prime}, C(t) y \cdot \nabla v_{m}\right)-i\left(\Delta v_{m}, C(t) y \cdot \nabla v_{m}\right)+\left(P_{m}\left[C(t) y \cdot \nabla v_{m}\right], C(t) y \cdot \nabla v_{m}\right) \\
+\operatorname{tr}(C(t))\left(v_{m}(t), C(t) y \cdot \nabla v_{m}\right)=\left(P_{m} f, C(t) y \cdot \nabla v_{m}\right)
\end{gathered}
$$

Note that $P_{m}^{2}=P_{m}$, then $\left(P_{m}\left[C(t) y \cdot \nabla v_{m}\right], C(t) y \cdot \nabla v_{m}\right)=\left|P_{m}\left[C(t) y \cdot \nabla v_{m}\right]\right|^{2}$ is real. Taking the double of the imaginary parts of both sides of (A.5), we have

$$
\begin{aligned}
& 2 \operatorname{Im}\left(v_{m}^{\prime}, C(t) y \cdot \nabla v_{m}\right)-2 \operatorname{Im} i\left(\Delta v_{m}, C(t) y \cdot \nabla v_{m}\right) \\
& \quad+2 \operatorname{tr}(C(t)) \operatorname{Im}\left(v_{m}(t), C(t) y \cdot \nabla v_{m}\right)=2 \operatorname{Im}\left(P_{m} f, C(t) y \cdot \nabla v_{m}\right) .
\end{aligned}
$$

Observing that $\operatorname{Im}(i z)=\operatorname{Re} z$, we modify (A.5), obtaining

$$
\begin{aligned}
& 2 \operatorname{Im}\left(v_{m}^{\prime}, C(t) y \cdot \nabla v_{m}\right)-2 \operatorname{Re}\left(\Delta v_{m}, C(t) y \cdot \nabla v_{m}\right) \\
& \quad+2 \operatorname{tr}(C(t)) \operatorname{Im}\left(v_{m}(t), C(t) y \cdot \nabla v_{m}\right)=2 \operatorname{Im}\left(P_{m} f, C(t) y \cdot \nabla v_{m}\right) .
\end{aligned}
$$

(i) Analysis of $2 \operatorname{Im}\left(v_{m}^{\prime}, C(t) y \cdot \nabla v_{m}\right)$. 
We have

$$
\frac{d}{d t}\left(v_{m}, C(t) y \cdot \nabla v_{m}\right)=\left(v_{m}^{\prime}, C(t) y \cdot \nabla v_{m}\right)+\left(v_{m}, C^{\prime}(t) y \cdot \nabla v_{m}\right)+\left(v_{m}, C(t) y \cdot \nabla v_{m}^{\prime}\right) .
$$

By Gauss' lemma,

$$
\int_{\Omega} \frac{\partial}{\partial y_{l}}\left(v_{m} C_{l k} y_{k} \overline{v_{m}^{\prime}}\right) d y=0
$$

that is,

$$
\int_{\Omega} \frac{\partial v_{m}}{\partial y_{l}} C_{l k} y_{k} \overline{v_{m}^{\prime}} d y+\int_{\Omega} v_{m} C_{l k} \delta_{l}^{k} \overline{v_{m}^{\prime}} d y+\int_{\Omega} v_{m} C_{l k} y_{k} \frac{\partial \overline{v_{m}^{\prime}}}{\partial y_{l}} d y=0
$$

Therefore, from (A.9), we get

$$
-\left(v_{m}, C(t) y \cdot \nabla v_{m}^{\prime}\right)=\operatorname{tr}(C)\left(v_{m}, v_{m}^{\prime}\right)+\left(C(t) y \cdot \nabla v_{m}, v_{m}^{\prime}\right) .
$$

Substituting (A.10) in (A.7), we obtain

$$
\begin{aligned}
& \left(v_{m}^{\prime}, C(t) y \cdot \nabla v_{m}\right)-\left(C(t) y \cdot \nabla v_{m}, v_{m}^{\prime}\right) \\
& \quad=\frac{d}{d t}\left(v_{m}, C(t) y \cdot \nabla v_{m}\right)-\left(v_{m}, C^{\prime}(t) y \cdot \nabla v_{m}\right)+\operatorname{tr}(C)\left(v_{m}, v_{m}^{\prime}\right) .
\end{aligned}
$$

Note that $z-\bar{z}=2 i \operatorname{Im} z$ and $-i(z-\bar{z})=2 \operatorname{Im} z$, which implies

$$
2 \operatorname{Im}\left(v_{m}^{\prime}, C(t) y \cdot \nabla v_{m}\right)=-i \frac{d}{d t}\left(v_{m}, C(t) y \cdot \nabla v_{m}\right)+i\left(v_{m}, C^{\prime}(t) y \cdot \nabla v_{m}\right)-i \operatorname{tr}(C)\left(v_{m}, v_{m}^{\prime}\right)
$$

Taking the real parts of both sides in the last equality, we obtain

$$
\begin{aligned}
2 \operatorname{Im}\left(v_{m}^{\prime}, C(t) y \cdot \nabla v_{m}\right)= & \operatorname{Im} \frac{d}{d t}\left(v_{m}, C(t) y \cdot \nabla v_{m}\right) \\
& -\operatorname{Im}\left(v_{m}, C^{\prime}(t) y \cdot \nabla v_{m}\right)+\operatorname{Im} \operatorname{tr}(C)\left(v_{m}, v_{m}^{\prime}\right) .
\end{aligned}
$$

From the projection (A.3), we have

$$
-v_{m}^{\prime}=-i \Delta v_{m}+P_{m}\left[C(t) y \cdot \nabla v_{m}\right]+\operatorname{tr}(C(t)) v_{m}-P_{m} f .
$$

Taking the inner product of both sides of (A.14) with $v_{m}$ and taking the imaginary parts of both sides, we obtain

$$
\operatorname{Im}\left(-v_{m}^{\prime}, v_{m}\right)=-\operatorname{Im} i\left(\Delta v_{m}, v_{m}\right)+\operatorname{Im}\left(P_{m}\left[C(t) y \cdot \nabla v_{m}, v_{m}\right]\right)-\operatorname{Im}\left(P_{m} f, v_{m}\right) .
$$

Observe that $\left(P_{m}\left[C(t) y \cdot \nabla v_{m}\right], v_{m}\right)=\left(C(t) y \cdot \nabla v_{m}, v_{m}\right)$, because $P_{m}$ is self-adjoint and $P_{m} v_{m}=v_{m}$. 
Therefore, from (A.15), it follows that

$$
\operatorname{Im}\left(-v_{m}^{\prime}, v_{m}\right)=-\operatorname{Re}\left(\Delta v_{m}, v_{m}\right)+\operatorname{Im}\left(C(t) y \cdot \nabla v_{m}, v_{m}\right)-\operatorname{Im}\left(P_{m} f, v_{m}\right) .
$$

From (A.16), we obtain

$$
\begin{aligned}
\operatorname{tr}(C(t)) \operatorname{Im}\left(v_{m}, v_{m}^{\prime}\right)= & \operatorname{tr}(C(t))\left\|v_{m}\right\|^{2}+\operatorname{tr}(C(t)) \operatorname{Im}\left(C(t) y \cdot \nabla v_{m}, v_{m}\right) \\
& -\operatorname{tr}(C(t)) \operatorname{Im}\left(P_{m} f, v_{m}\right)
\end{aligned}
$$

We modify (A.13), by means of (A.17) and obtain

$$
\begin{aligned}
2 \operatorname{Im}\left(v_{m}^{\prime}, C(t) y \cdot \nabla v_{m}\right)= & \operatorname{Im} \frac{d}{d t}\left(v_{m}, C(t) y \cdot \nabla v_{m}\right)-\operatorname{Im}\left(v_{m}, C^{\prime}(t) y \cdot \nabla v_{m}\right) \\
& +\operatorname{tr}(C(t))\left\|v_{m}\right\|^{2}+\operatorname{tr}(C(t)) \operatorname{Im}\left((t) y \cdot \nabla v_{m}, v_{m}\right) \\
& -\operatorname{tr}(C(t)) \operatorname{Im}\left(P_{m} f, v_{m}\right)
\end{aligned}
$$

From the second estimate, we have

$$
\begin{aligned}
2 \operatorname{Re}\left(\nabla v_{m}(t) \cdot C(t) y,-\Delta v_{m}(t)\right)= & 2 \operatorname{Re}\left(\nabla v_{m}(t) \cdot C(t) y, \nabla v_{m}(t)\right) \\
& -\operatorname{tr}(C(t))\left\|v_{m}(t)\right\|^{2}-\int_{\Gamma} C(t) y \cdot v\left|\frac{\partial v_{m}}{\partial \nu}\right|^{2} d \Gamma .
\end{aligned}
$$

Substituting (A.18) and (A.19) in (A.6), it follows that

$$
\begin{aligned}
&-\int_{\Gamma} C(t) y \cdot v\left|\frac{\partial v_{m}}{\partial \nu}\right|^{2} d \Gamma+2 \operatorname{Re}\left(\nabla v_{m}(t) \cdot C(t) y, \nabla v_{m}(t)\right)-\operatorname{tr}(C(t))\left\|v_{m}(t)\right\|^{2} \\
&= 2 \operatorname{Im}\left(P_{m} f, \nabla v_{m}(t) \cdot C(t) y\right)+\operatorname{tr}(C(t)) \operatorname{Im}\left(P_{m} f, v_{m}\right) \\
&-\operatorname{tr}(C(t)) \operatorname{Im}\left(v_{m}(t), C(t) y \cdot \nabla v_{m}\right)-\operatorname{tr}(C(t))\left\|v_{m}\right\|^{2} \\
&-\frac{d}{d t} \operatorname{Im}\left(v_{m}, C(t) y \cdot \nabla v_{m}\right)+\operatorname{Im}\left(v_{m}, C^{\prime}(t) y \cdot \nabla v_{m}\right)
\end{aligned}
$$

\section{Acknowledgments}

We thank Professor Luis Adauto Medeiros for drawing our attention to this question and for his assistance while developing the project. We also thank Professor M. Milla Miranda for the constructive conversation about the subject of the paper. We would also like to thank the referee for the valuable suggestions which have improved the text comprehension.

\section{References}

[1] H. Brézis, Opérateurs Maximaux Monotones et Semi-groupes de Contractions dans les espaces de Hilbert, North-Holland Mathematics Studies, no. 5. Notas de Matemática (50), North-Holland, Amsterdam, 1973.

[2] P. Cannarsa, G. Da Prato, and J.-P. Zolésio, The damped wave equation in a moving domain, Journal of Differential Equations 85 (1990), no. 1, 1-16. 
[3] J. Cooper and C. Bardos, A nonlinear wave equation in a time dependent domain, Journal of Mathematical Analysis and Applications 42 (1973), no. 1, 29-60.

[4] C. He and L. Hsiao, Two-dimensional Euler equations in a time dependent domain, Journal of Differential Equations 163 (2000), no. 2, 265-291.

[5] A. Inoue, Sur $\square u+u^{3}=f$ dans un domaine noncylindrique, Journal of Mathematical Analysis and Applications 46 (1974), no. 3, 777-819.

[6] G. Lebeau, Contrôle de l'équation de Schrödinger, Journal de Mathématiques Pures et Appliquées 71 (1992), no. 3, 267-291.

[7] J.-L. Lions, Problèmes aux Limites dans Les Équations aux Dérivées Partielles, Deuxième édition. Séminaire de Mathématiques Supérieures, no. 1 (Été, 1962), Les Presses de l'Université de Montréal, Montreal, 1965, see also Oeuvres Choìsis de Jacques Lois Lions, Vol. I (2003) pp. 431-476 SMAI, EDP Sciences Paris - France.

[8] Contrôlabilité Exacte, Perturbations et Stabilisation de Systèmes Distribués. Tome 1, Contrôlabilité Exacte, Research in Applied Mathematics, vol. 8, Masson, Paris, 1988.

[9] E. Machtyngier, Contrôlabilité exacte et stabilisation frontière de l'équation de Schrödinger, Comptes Rendus de l'Académie des Sciences. Série I. Mathématique 310 (1990), no. 12, 801806.

[10] M. M. Miranda and L. A. Medeiros, Contrôlabilité exacte de l'équation de Schrödinger dans des domaines non cylindriques, Comptes Rendus de l'Académie des Sciences 319 (1994), no. 7, 685689.

[11] R. Temam, Navier-Stokes Equations, Theory and Numerical Analysis, Studies in Mathematics and Its Applications, vol. 2, North-Holland, Amsterdam, 1979.

G. O. Antunes: Instituto de Matemática e Estatística, Universidade do Estado do Rio de Janeiro, Rio de Janeiro 20550-900, Brazil

E-mail address: gladson@uerj.br

M. D. G. da Silva: Instituto de Matemática, Universidade Federal do Rio de Janeiro,

Rio de Janeiro 21941-590, Brazil

E-mail address: darci@im.ufrj.br

R. F. Apolaya: Instituto de Matemática, Universidade Federal Fluminense, 24020-140 Niterói,

Rio de Janeiro, Brazil

E-mail address: ganrifa@vm.uff.br 


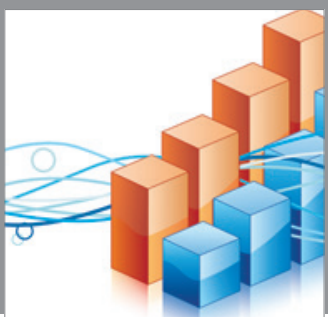

Advances in

Operations Research

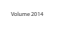

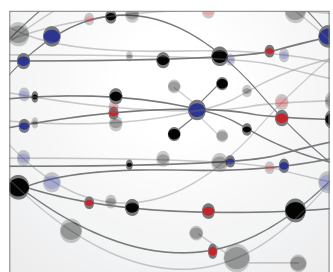

\section{The Scientific} World Journal
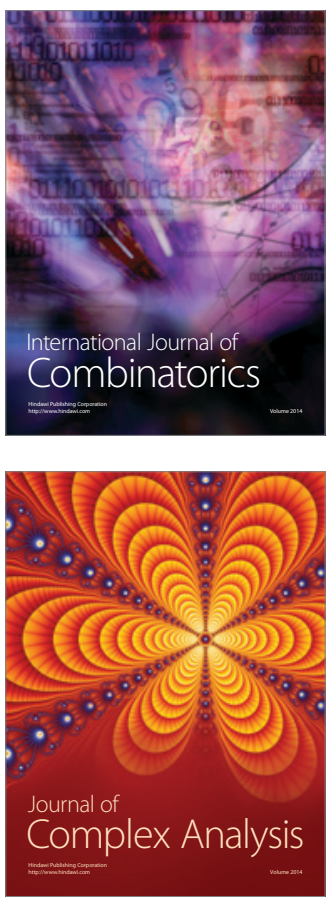

International Journal of

Mathematics and

Mathematical

Sciences
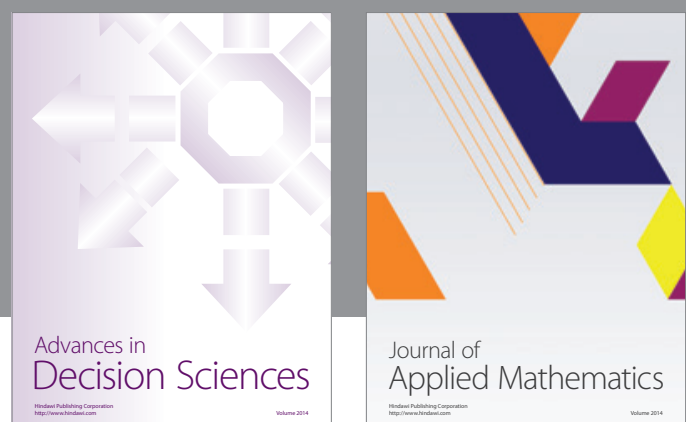

Journal of

Applied Mathematics
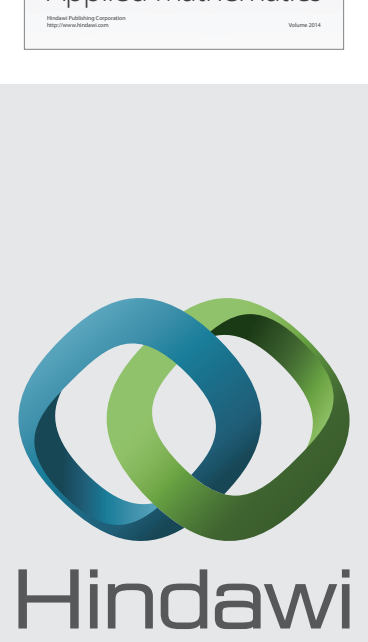

Submit your manuscripts at http://www.hindawi.com
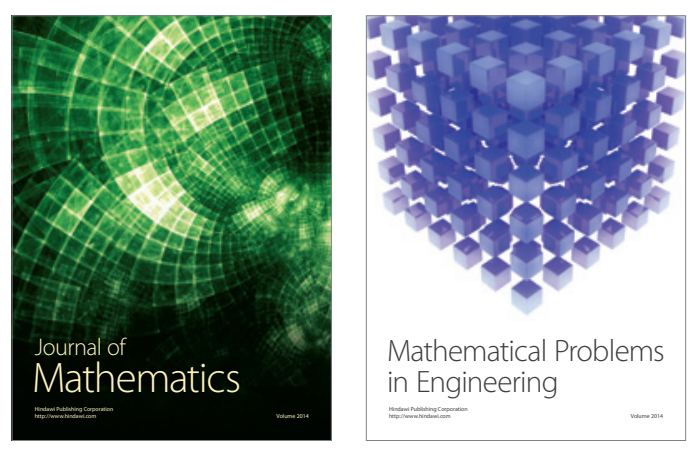

Mathematical Problems in Engineering
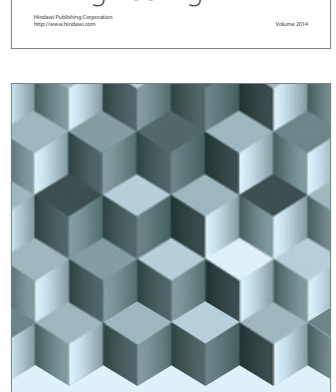

Journal of

Function Spaces
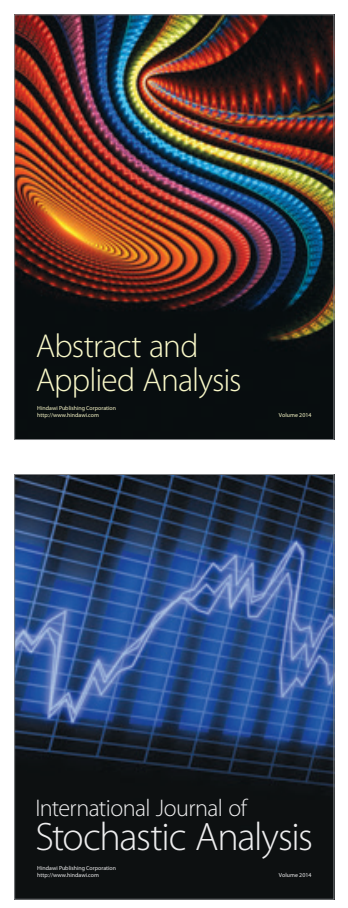

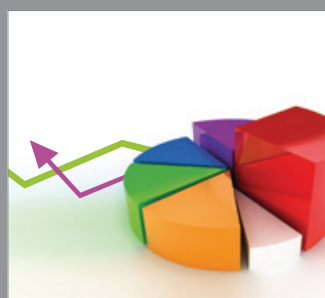

ournal of

Probability and Statistics

Promensencen
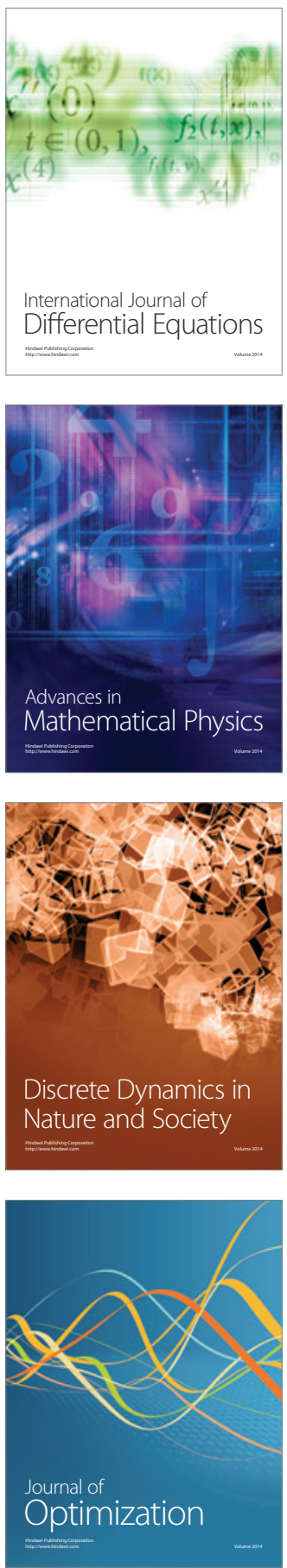